\title{
On the Rigidity of Moduli of Curves in Arbitrary Characteristic
}

\author{
Barbara Fantechi ${ }^{1}$ and Alex Massarenti ${ }^{2, *}$ \\ ${ }^{1}$ SISSA, via Bonomea 265, 34136 Trieste, Italy and ${ }^{2} \mathrm{UFF}$, Rua Mário \\ Santos Braga, 24020-140 Niterói, Rio de Janeiro, Brazil \\ *Correspondence to be sent to: e-mail: alexmassarenti@id.uff.br
}

The stack $\overline{\mathcal{M}}_{g, n}$ of stable curves and its coarse moduli space $\bar{M}_{g, n}$ are defined over $\mathbb{Z}$, and therefore over any field. Over an algebraically closed field of characteristic zero, in [11] Hacking showed that $\overline{\mathcal{M}}_{g, n}$ is rigid (a conjecture of Kapranov), in [2, 23] Bruno and Mella for $g=0$, and the second author for $g \geq 1$ showed that its automorphism group is the symmetric group $S_{n}$, permuting marked points unless $(g, n) \in\{(0,4),(1,1),(1,2)\}$. The methods used in the papers above do not extend to positive characteristic. We show that in characteristic $p>0$, the rigidity of $\overline{\mathcal{M}}_{g, n}$, with the same exceptions as over $\mathbb{C}$, implies that its automorphism group is $S_{n}$. We prove that, over any field, $\bar{M}_{0, n}$ is rigid and deduce that, over any field, $\operatorname{Aut}\left(\bar{M}_{0, n}\right) \cong S_{n}$ for $n \geq 5$. Going back to characteristic zero, we prove that for $g+n>4$, the coarse moduli space $\bar{M}_{g, n}$ is rigid, extending a result of Hacking who had proven it has no locally trivial deformations. Finally, we show that $\bar{M}_{1,2}$ is not rigid, although it does not admit locally trivial deformations, by explicitly computing his Kuranishi family.

\section{Introduction}

The stack $\overline{\mathcal{M}}_{g, n}$ parametrizing Deligne-Mumford stable curves and its coarse moduli space $\bar{M}_{g, n}$ are among the most fascinating and widely studied objects in algebraic geometry. A remarkable property of $\overline{\mathcal{M}}_{g, n}$ is that it is smooth and proper over $\operatorname{Spec}(\mathbb{Z})$, and 
thus $\overline{\mathcal{M}}_{g, n}^{R}$ is defined over any commutative ring $R$ via base change

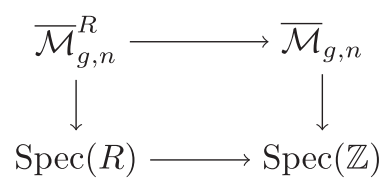

By [18] the formation of the coarse moduli space is compatible with flat base change; we write $\bar{M}_{g, n}^{R}$ for the coarse moduli scheme of $\overline{\mathcal{M}}_{g, n}^{R}$.

The symmetric group $S_{n}$ acts via permutations of the marked points on $\bar{M}_{g, n}^{R}$ and $\overline{\mathcal{M}}_{g, n}^{R}$ for every ring $R$. The biregular automorphisms of the moduli space $M_{g, n}$, and of its Deligne-Mumford compactification $\bar{M}_{g, n}$ have been studied in a series of papers $[2,9,21-26,33]$.

It is known that, with a short and explicit list of exceptions, over the field of complex numbers both the automorphism groups of the stack and of the coarse moduli space are isomorphic to $S_{n}$, see Appendix for details.

In Section 2 we first study how automorphisms of a scheme behave with respect to field extensions, and then, given a scheme $X \rightarrow \operatorname{Spec}(A)$ over a local ring $A$ with residue field $K$ and generic point $\xi \in \operatorname{Spec}(A)$, we show how the infinitesimal rigidity of $X^{K}$ plays a fundamental role in lifting automorphisms of $X^{K}$ to automorphisms of $X_{\xi}$.

Finally, in Section 7 we apply these results together with the rigidity results in Section 4 to $\bar{M}_{0, n}^{K}$, with $K$ a field of positive characteristic and $A=W(K)$ the ring of Witt vector over $K$, in order to compute the automorphism group of $\bar{M}_{0, n}^{K}$. The main results on the automorphism groups in Proposition 5.4, Theorem 7.3, and Appendix can be summarized as follows:

Theorem 1.1. Let $K$ be any field. Then

$$
\operatorname{Aut}\left(\bar{M}_{0, n}^{K}\right) \cong S_{n}
$$

for any $n \geq 5$, and $\operatorname{Aut}\left(\bar{M}_{0,4}^{K}\right) \cong \operatorname{PGL}(2)$. If $\operatorname{char}(K) \neq 2,2 g-2+n \geq 3,(g, n) \neq(2,1)$ and $n \geq 1$ then

$$
\operatorname{Aut}\left(\overline{\mathcal{M}}_{g, n}^{K}\right) \cong \operatorname{Aut}\left(\bar{M}_{g, n}^{K}\right) \cong S_{n}
$$

Over any field $K$ of characteristic zero we have that $\operatorname{Aut}\left(\overline{\mathcal{M}}_{g}^{K}\right) \cong \operatorname{Aut}\left(\bar{M}_{g}^{K}\right)$ for any $g \geq 2$, and $\operatorname{Aut}\left(\overline{\mathcal{M}}_{2,1}^{K}\right) \cong \operatorname{Aut}\left(\bar{M}_{2,1}^{K}\right)$ are trivial . 
Finally, if $K$ is an algebraically closed field with $\operatorname{char}(K) \neq 2,3$ we have

$$
\operatorname{Aut}\left(\bar{M}_{1,2}^{K}\right) \cong\left(K^{*}\right)^{2}
$$

while Aut $\left(\overline{\mathcal{M}}_{1,2}^{K}\right)$ is trivial, $\operatorname{Aut}\left(\overline{\mathcal{M}}_{1,1}^{K}\right) \cong K^{*}$, and $\operatorname{Aut}\left(\bar{M}_{1,1}^{K}\right) \cong \operatorname{PGL}(2)$.

The rigidity of the moduli stack of stable curves is interesting in its own sake, as a special case of the following conjecture proposed by Kapranov in [16]:

Conjecture. Let $X$ be a smooth scheme, and let $X_{1}$ be the moduli space of deformations of $X, X_{2}$ the moduli space of deformations of $X_{1}$, and so on. Then $X_{\operatorname{dim}(X)}$ is rigid, that is it does not have infinitesimal deformations.

Over a field of characteristic zero the rigidity of the stack $\overline{\mathcal{M}}_{g, n}$ has been proven by Hacking in [11, Theorem 2.1]. Furthermore, by [11, Theorems 2.3], the coarse moduli space $\bar{M}_{g, n}$ does not have non-trivial, locally trivial deformations if $(g, n) \notin$ $\{(1,2),(2,0),(2,1),(3,0)\}$.

In Section 3, we review Hacking's proof and explain why it fails to extend to positive characteristic; the main problem is the lack of a suitable version of Kodaira vanishing. We then show that the requested vanishing can be proven directly in genus zero, thus establishing the rigidity of $\bar{M}_{0, n}^{K}=\overline{\mathcal{M}}_{0, n}^{K}$ for any field $K$, and hence deducing that its automorphism group if $S_{n}$ for $n \geq 5$.

Finally, in Section 6 we address a question Hacking left open, namely the rigidity of the scheme $\bar{M}_{g, n}^{K}$ when $K$ is an algebraically closed field of characteristic zero. We show that the Kuranishi family of $\bar{M}_{1,2}^{K}$ is non-singular of dimension 6 and that the general deformation is smooth; for $g+n>4$ we show that $\bar{M}_{g, n}^{K}$ is rigid. This provides an interesting example of a rigid scheme whose singularities are not locally rigid. The main result in [11] together with Theorems 4.1, 6.5, 5.8, 6.13 can be summarized in the following statement:

Theorem 1.2. Over any field $K$ the moduli space $\bar{M}_{0, n}^{K}$ is rigid for any $n \geq 3$. If $K$ is an algebraically closed field of characteristic zero then the stack $\overline{\mathcal{M}}_{g, n}^{K}$ is rigid, and its coarse moduli space $\bar{M}_{g, n}^{K}$ is rigid if $g+n>4$ and does not have locally trivial deformations if $g+n \geq 4$.

Finally, the coarse moduli space $\bar{M}_{1,2}^{K}$ does not have locally trivial deformations, while its family of first-order infinitesimal deformations is non-singular of dimension 6 and the general deformation is smooth. 
Finally, we would like to mention that recently the non-commutative rigidity of $\overline{\mathcal{M}}_{g, n}$, in characteristic zero, has been proven by means of the vanishing of the second Hochschild cohomology by Okawa and Sano in [29].

\section{Automorphisms and Rigidity}

First of all, we need some elementary results on the behavior of automorphisms with respect to field extensions. In the following by orbifold, we always mean a non-singular Deligne-Mumford stack with trivial general stabilizer; this assumption implies that the automorphism groupoid is rigid, hence can be identified with a group.

Remark 2.1. Let $K \subseteq L$ be a field extension, $X_{K}$ a scheme, or an orbifold, over $K$ and $X_{L}:=X_{K} \times_{\operatorname{Spec}(K)} \operatorname{Spec}(L)$. Any morphism $f_{K}: X_{K} \rightarrow X_{K}$ over $K$ induces a unique morphism $f_{L}: X_{L} \rightarrow X_{L}$ over $L$ by the universal property of the fiber product applied to the following commutative diagram.

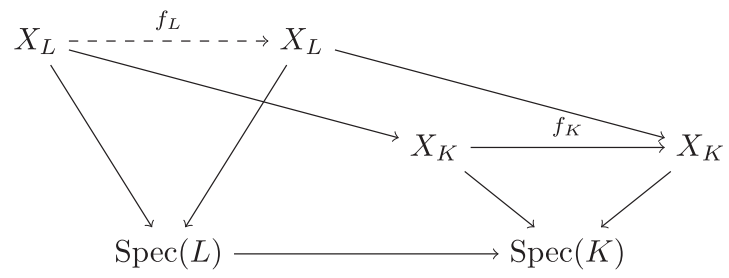

Lemma 2.2. Let $K \subseteq L$ be a field extension, $X_{K}$ a scheme over $K$ and $X_{L}:=X_{K} \times_{\operatorname{Spec}(K)}$ $\operatorname{Spec}(L)$. Then, there exists an injective morphism of groups

$$
\chi: \operatorname{Aut}\left(X_{K}\right) \rightarrow \operatorname{Aut}\left(X_{L}\right)
$$

Proof. The existence of the morphism $\chi$ is an immediate consequence of Remark 2.1. To prove injectivity, let $\phi_{K} \in \operatorname{Aut}\left(X_{K}\right)$ be an automorphism such that the induced automorphism $\phi_{L} \in \operatorname{Aut}\left(X_{L}\right)$ is the identity; we need to show the $\phi_{K}$ is also the identity. Being the identity is local in the étale topology, so we may assume $X=\operatorname{Spec}(A)$ with $A$ a $K$ algebra and $\phi_{K}$ induced by $f \in \operatorname{Aut}(A)$. We have that $f-\operatorname{Id}_{A}$ is a $K$-linear automorphism of $A$ which is zero when tensored with $L$, therefore it is zero.

In the rest of this section $A$ will be a local ring with maximal ideal $\mathfrak{m}$ and residue field $K$, which is complete in the m-adic topology. Let $X$ be a Deligne-Mumford stack and $f: X \rightarrow \operatorname{Spec}(A)$ be a proper, flat morphism. Let $A_{n}:=A / \mathfrak{m}^{n+1}$, and $X_{n}:=X \times_{\operatorname{Spec}(A)}$ $\operatorname{Spec}\left(A_{n}\right)$. Write $\operatorname{Aut}\left(X_{n} / A_{n}\right)$ for the automorphism group of $X_{n}$ over $\operatorname{Spec}\left(A_{n}\right)$. Let $\xi$ be the 
generic point of $\operatorname{Spec}(A)$ and $X_{\xi}$ the corresponding fiber of $X$. Assume that $X_{0}$ is either a scheme or an orbifold.

Lemma 2.3. Assume that $X_{0}$ is smooth, then for every $n \geq 0$ there is an exact sequence of sets

$$
0 \rightarrow H^{0}\left(X_{0}, T_{X_{0}}\right) \otimes \mathfrak{m}^{n} / \mathfrak{m}^{n+1} \rightarrow \operatorname{Aut}\left(X_{n+1}\right) \rightarrow \operatorname{Aut}\left(X_{n}\right) \rightarrow H^{1}\left(X_{0}, T_{X_{0}}\right) \otimes \mathfrak{m}^{n} / \mathfrak{m}^{n+1}
$$

where $\alpha: \operatorname{Aut}\left(X_{n+1}\right) \rightarrow \operatorname{Aut}\left(X_{n}\right)$ is the restriction map. In particular, if $H^{i}\left(X_{0}, T_{X_{0}}\right)=0$ for $i=0,1$, then $\operatorname{Aut}(X / A)$ is formally étale over $A$.

Proof. By exact sequence we mean that an element $f \in \operatorname{Aut}\left(X_{n} / A_{n}\right)$ is in the image of $\alpha$ if and only if its image in $H^{1}\left(X_{0}, T_{X_{0}}\right) \otimes \mathfrak{m}^{n} / \mathfrak{m}^{n+1}$ is zero, and in this case the set of inverse images is naturally a principal homogeneous space for the group $H^{0}\left(X_{0}, T_{X_{0}}\right) \otimes \mathfrak{m}^{n} / \mathfrak{m}^{n+1}$. The statement follows from [14, Proposition III. 2.2.2] by observing that since $X$ is smooth over $\operatorname{Spec}(A)$ the cotangent complex is $L_{X / A}=\Omega_{X / A}$.

Remark 2.4. If we only assume that $X_{0}$ is reduced, Lemma 2.3 still holds with $\operatorname{Ext}^{1}\left(\Omega_{X_{0}}, \mathcal{O}_{X_{0}}\right)$ in place of $H^{1}\left(X_{0}, T_{X_{0}}\right)$; this is an easy and well-known computation.

Definition 2.5. Let $X$ be a scheme over a field. We will say it is rigid if it has no nontrivial infinitesimal deformations; if $X$ is smooth, this is equivalent to $H^{1}\left(X, T_{X}\right)=0$ and if $X$ is generically reduced this is equivalent to $\operatorname{Ext}^{1}\left(\Omega_{X}, \mathcal{O}_{X}\right)=0$.

Theorem 2.6. Assume that $X \rightarrow \operatorname{Spec}(A)$ is a proper, separated morphism of schemes, that $X_{0} \rightarrow \operatorname{Spec}(A / \mathfrak{m})$ is generically reduced and rigid, and that $H^{0}\left(X_{\xi}, T_{X_{\xi}}\right)=0$. Then, there is a natural injective morphism of groups

$$
\chi: \operatorname{Aut}\left(X_{0}\right) \rightarrow \operatorname{Aut}\left(X_{\xi}\right)
$$

Proof. We have a family of infinitesimal deformations $\left\{X_{n}\right\}_{n \geq 0}$ where $X_{n}$ is a scheme over $A_{n}$. Since $X_{0}$ is rigid we have $H^{1}\left(X_{0}, T_{X_{0}}\right)=0$, and by semi-continuity $H^{0}\left(X_{\xi}, T_{X_{\xi}}\right)=0$ implies $H^{0}\left(X_{0}, T_{X_{0}}\right)=0$.

Lemma 2.3 implies that any automorphism of $X_{n}$ lifts to a unique automorphism of $X_{n+1}$. Therefore, starting with an automorphism $\phi:=\phi_{0} \in \operatorname{Aut}\left(X_{0}\right)$ we may construct a family of automorphisms $\phi_{\bullet}=\left\{\phi_{n}\right\}_{n \geq 0}$, where $\phi_{n}$ is an automorphism of $X_{n}$. The direct limit

$$
\widehat{X}=\underset{n}{\lim } X_{n} \rightarrow \operatorname{Spf}(A)
$$


is a formal scheme over the formal spectrum $\operatorname{Spf}(A)$ of $A$. By [7, Section 8.1.5] $\phi$. induces a unique automorphism $\widehat{\phi}: \widehat{X} \rightarrow \widehat{X}$, and by [7, Corollary 8.4.7] $\widehat{\phi}$ in turn induces an unique automorphism $\bar{\phi}$ of $X \rightarrow \operatorname{Spec}(A)$. Let $X_{\xi}$ be the general fiber of $X \rightarrow \operatorname{Spec}(A)$. By taking the restriction of $\bar{\phi}$ to $X_{\xi}$ we get a morphism of groups

$$
\begin{array}{cl}
\chi: \operatorname{Aut}\left(X_{0}\right) & \longrightarrow \operatorname{Aut}\left(X_{\xi}\right) \\
\phi & \longmapsto \bar{\phi}_{\mid X_{\xi}} .
\end{array}
$$

Clearly if $\phi$ and $\psi$ are two automorphisms of $X_{0}$ inducing the same automorphism of $X_{\xi}$ then $\bar{\phi}=\bar{\psi}$ because $\xi$ is the general point of $\operatorname{Spec}(A)$ and $X$ is separated. In particular $\bar{\phi}$ and $\bar{\psi}$ coincide on the special fiber $X_{0}$, that is, $\phi=\psi$. So, the morphism $\chi$ is injective.

We will need the following result in order to study the automorphism group of the stack $\overline{\mathcal{M}}_{g, n}$.

Proposition 2.7. Let $\mathcal{X}$ be a separated orbifold over an affine scheme $S$ and let $X$ be its coarse moduli space. Then, there exists an injective morphism of groups

$$
\chi: \operatorname{Aut}(\mathcal{X} / S) \rightarrow \operatorname{Aut}(X / S)
$$

Proof. Since the natural map $\pi: \mathcal{X} \rightarrow X$ is universal for morphisms in schemes for any $\phi \in \operatorname{Aut}(\mathcal{X})$, we get a unique $\tilde{\phi} \in \operatorname{Aut}(X)$ commuting with $\pi$. This correspondence induces the morphism $\chi$. The orbifold condition implies that there is an open substack $U$ of $\mathcal{X}$ which is isomorphic to its image in $X$; if $\phi \in \operatorname{Aut}(X / S)$ satisfies $\chi(\phi)=\operatorname{Id}_{X}$, it follows that $\left.\phi\right|_{U}=\operatorname{Id}_{U}$ and by separateness this implies that $\phi=\operatorname{Id}_{\mathcal{X}}$.

\section{A Review of Hacking's Proof of Rigidity in Characteristic Zero}

We begin this section working over the integers. We will point out when we pass to characteristic zero. Let $X$ be a smooth algebraic stack, $i: D \rightarrow X$ the closed embedding of a normal crossing divisor, nc for short, $v: D^{v} \rightarrow D$ its normalization; by definition of $\mathrm{nc}, D^{v}$ is smooth.

The composition $\bar{v}:=i \circ v: D^{v} \rightarrow X$ is an immersion, that is, the differential has maximum rank at every point; hence it has a normal line bundle $\mathcal{N}$, defined by the exact sequence

$$
0 \mapsto T_{D^{v}} \rightarrow \bar{\nu}^{*}\left(T_{X}\right) \rightarrow \mathcal{N} \rightarrow 0
$$


The vector bundle of logarithmic differentials $T_{X}(-\log D)$ is defined by the exact sequence

$$
0 \mapsto T_{X}(-\log D) \rightarrow T_{X} \rightarrow \bar{v}_{*}(\mathcal{N}) \rightarrow 0
$$

Let $\pi: \mathcal{U}_{g, n} \rightarrow \overline{\mathcal{M}}_{g, n}$ be the universal curve. Let $\mathcal{B}$ be the boundary of $\overline{\mathcal{M}}_{g, n}$; it is a normal crossing divisor. Its normalization $\mathcal{B}^{v}$ is the disjoint union of products $\overline{\mathcal{M}}_{g_{1}, n_{1}+1} \times$ $\overline{\mathcal{M}}_{g_{2}, n_{2}+1}$ with $g_{1}+g_{2}=g$ and $n_{1}+n_{2}=n$, together with $\overline{\mathcal{M}}_{g-1, n+2}$ by [19, Corollary 3.9]. The restriction of $\mathcal{N}$ to each of these components is $p_{1}^{*} \psi_{n_{1}+1}^{\vee} \otimes p_{2}^{*} \psi_{n_{2}+1}^{\vee}$ and $\psi_{n+1}^{\vee} \otimes \psi_{n+2}^{\vee}$, respectively. Let $\Sigma \subset \mathcal{U}_{g, n}$ be the union of the images of the $n$ sections of $\pi$, this is an nc divisor.

Lemma 3.1. [11, Lemma 3.1] There is an isomorphism $T_{\overline{\mathcal{M}}_{g, n}}(-\log \mathcal{B}) \rightarrow R^{1} \pi_{*}\left(\omega_{\pi}(\Sigma)^{\vee}\right)$.

Corollary 3.2. For every $k \geq 0$ we have $H^{k}\left(\mathcal{U}_{g, n} \omega_{\pi}(\Sigma)^{\vee}\right)=H^{k-1}\left(\overline{\mathcal{M}}_{g, n}, T_{\overline{\mathcal{M}}_{g, n}}(-\log \mathcal{B})\right)$.

Proof. Since all other push-forwards of $\omega_{\pi}(\Sigma)^{\vee}$ vanish, it follows from Leray's spectral sequence.

Lemma 3.3. [19, Theorem 4] The pull-back under the natural isomorphism $\overline{\mathcal{M}}_{g, n+1} \rightarrow$ $\mathcal{U}_{g, n}$ of the line bundle $\omega_{\pi}(\Sigma)$ is $\psi_{n+1}$.

Putting these results together, Hacking is able to reduce the rigidity of $\overline{\mathcal{M}}_{g, n}$ to vanishing of cohomologies of line bundles.

Proposition 3.4. Assume that $H^{2}\left(\overline{\mathcal{M}}_{g, n+1}, \psi_{n+1}^{\vee}\right)=0$, and that $H^{1}\left(\mathcal{B}^{v}, \mathcal{N}\right)=0$. Then $\overline{\mathcal{M}}_{g, n}$ is rigid. More generally, if $H^{i+1}\left(\overline{\mathcal{M}}_{g, n+1}, \psi_{n+1}^{\vee}\right)=H^{i}\left(\mathcal{B}^{v}, \mathcal{N}\right)=0$ then $H^{i}\left(\overline{\mathcal{M}}_{g, n}, T_{\overline{\mathcal{M}}_{g, n}}\right)=0$.

\subsection{Characteristic zero part}

By [17, Theorem 0.4] the $\mathbb{Q}$-line bundle $p_{*} \omega_{\pi}(\Sigma)$ is nef and big. In [11, Theorem 3.2] Hacking proves that, over a field $K$ of characteristic zero,

$$
H^{i+1}\left(\mathcal{U}_{g, n}, \omega_{\pi}(\Sigma)^{\vee}\right) \cong H^{i}\left(\overline{\mathcal{M}}_{g, n}, T_{\overline{\mathcal{M}}_{g, n}}(-\log (\mathcal{B}))\right)=0
$$

for any $i<\operatorname{dim}\left(\overline{\mathcal{M}}_{g, n}\right)$. Furthermore, by [11, Corollary 4.4] the $\mathbb{Q}$-line bundle on the coarse moduli space of $\mathcal{B}^{v}$ defined by $\mathcal{N}^{\vee}$ is nef and big on each component. Then,

$$
H^{i}\left(\overline{\mathcal{M}}_{g, n}, v_{*} \mathcal{N}\right)=0
$$


for $i<\operatorname{dim}(\mathcal{B})$. Finally, combining the vanishings (3.1) and (3.2) Hacking proves that

$$
H^{i}\left(\overline{\mathcal{M}}_{g, n}, T_{\overline{\mathcal{M}}_{g, n}}\right)=0
$$

for $i<\operatorname{dim}\left(\overline{\mathcal{M}}_{g, n}\right)-1$. In particular $H^{1}\left(\overline{\mathcal{M}}_{g, n}, T_{\overline{\mathcal{M}}_{g, n}}\right)=0$, that is $\overline{\mathcal{M}}_{g, n}$ is rigid. Both the vanishings (3.1) and (3.2) are consequences of [11, Theorem A.1] which is a version of Kodaira vanishing for proper and smooth Deligne-Mumford stacks. This theorem derives from the Kodaira vanishing theorem for proper normal varieties. Therefore it holds only in characteristic zero. At the best of our knowledge, the closest result to Kodaira vanishing in positive characteristic is the Deligne-Illusie vanishing [5]. However, such result works for ample line bundle while in positive characteristic the involved psi-classes are just semi-ample [17].

\section{Rigidity of $\bar{M}_{0, n}$ in Positive Characteristic}

In this section, we study the $g=0$ case; any $n$-pointed stable genus-zero curve is automorphisms free, thus the coarse moduli space $\bar{M}_{0, n}$ is smooth and coincides with the stack. In [15] Kapranov constructed $\bar{M}_{0, n}$ via a sequence of blow-ups of $\mathbb{P}^{n-3}$ along linear spaces in order of increasing. The blow-up morphism $f: \bar{M}_{0, n} \rightarrow \mathbb{P}^{n-3}$ is induced by a psi-class $\psi_{n}$.

In [15] Kapranov works, for sake of simplicity, on an algebraically closed field of characteristic zero. On the other hand, Kapranov's arguments are purely algebraic and his description works over $\operatorname{Spec}(\mathbb{Z})$.

Theorem 4.1. Let $K$ be a field, $n \geq 3$. Then, except for $i=0$ and $n=4$, we have

$$
H^{i}\left(\bar{M}_{0, n}, T_{\bar{M}_{0, n}}\right)=0 \text { for all } i
$$

Hence, $\bar{M}_{0, n}$ is rigid for any $n \geq 3$.

Proof. The case $n=3$ is obvious since the tangent bundle is zero, hence we may assume $n \geq 4$. By Proposition 3.4 it is enough to prove that

- $H^{i}\left(\mathcal{U}_{0, n+1}, \omega_{\pi}(\Sigma)^{\vee}\right)=0$,

- for all $n_{1}+n_{2}=n$ with $n_{1}, n_{2} \geq 2$, for all $i_{1}+i_{2}=i$ one has

$$
H^{i_{1}}\left(\bar{M}_{0, n_{1}+1}, \psi_{n_{1}}^{\vee}\right) \otimes H^{i_{2}+1}\left(\bar{M}_{0, n_{1}+1}, \psi_{n_{2}+1}^{\vee}\right)=0 .
$$


Let us consider the line bundle $\omega_{\pi}(\Sigma)$ over $\mathcal{U}_{0, n} \cong \bar{M}_{0, n+1}$. By Lemma $3.3 \omega_{\pi}(\Sigma)$ identifies with $\psi_{n+1}$. Recall that we have a birational morphism

$$
f: \bar{M}_{0, n+1} \rightarrow \mathbb{P}^{n-2}
$$

such that $f^{*} \mathcal{O}_{\mathbb{P}^{n-2}}(1) \cong \omega_{\pi}(\Sigma) \cong \psi_{n+1}$ given by the Kapranov's blow-up construction. Now, by [12, Theorem 5.1] we have that $H^{i}\left(\mathbb{P}^{n-2}, \mathcal{O}_{\mathbb{P}^{n-2}}(-1)\right)=0$ for $i<n-2$. Furthermore we have $H^{0}\left(\mathbb{P}^{n-2}, \mathcal{O}_{\mathbb{P} n-2}(-1)\right)=0$, and by Serre duality $H^{n-2}\left(\mathbb{P}^{n-2}, \mathcal{O}_{\mathbb{P}^{n-2}}(-1)\right) \cong$ $H^{0}\left(\mathbb{P}^{n-2}, \mathcal{O}_{\mathbb{P}} n-2(2-n)\right)=0$ for $n \geq 3$. Then $H^{i}\left(\mathbb{P}^{n-2}, \mathcal{O}_{\mathbb{P}^{n-2}}(-1)\right)=0$ for any $i$. By the projection formula we get

$$
f_{*}\left(\psi_{n+1}^{\vee}\right)=f_{*}\left(\mathcal{O}_{\bar{M}_{0, n+1}} \otimes f^{*} \mathcal{O}_{\mathbb{P}} n-2(-1)\right) \cong f_{*}\left(\mathcal{O}_{\bar{M}_{0, n+1}}\right) \otimes \mathcal{O}_{\mathbb{P}} n-2(-1)
$$

Since $f$ is a birational morphism between smooth varieties, we have $f_{*}\left(\mathcal{O}_{\bar{M}_{0, n+1}}\right) \cong \mathcal{O}_{\mathbb{P} n-2}$; hence $f_{*}\left(\psi_{n+1}^{\vee}\right) \cong \mathcal{O}_{\mathbb{P}^{n-2}}(-1)$.

Moreover since $f$ is a sequence of blow-ups of smooth varieties with smooth centers, we have $R^{i} f_{*} \psi_{n+1}^{\vee}=0$ for $i>0$, and by the Leray spectral sequence we get $H^{i}\left(\bar{M}_{0, n+1}, \psi_{n+1}^{\vee}\right) \cong H^{i}\left(\mathbb{P}^{n-2}, f_{*} \psi_{n+1}^{\vee}\right)$ for any $i \geq 0$. Therefore,

$$
H^{i}\left(\bar{M}_{0, n+1}, \psi_{n+1}^{\vee}\right) \cong H^{i}\left(\mathbb{P}^{n-2}, \mathcal{O}_{\mathbb{P}^{n-2}}(-1)\right)=0 \text { for any } i
$$

Summing up we proved the following vanishings:

$$
H^{i+1}\left(\bar{M}_{0, n+1}, \psi_{n+1}^{\vee}\right) \cong H^{i+1}\left(\bar{M}_{0, n+1}, \omega_{\pi}(\Sigma)^{\vee}\right) \cong H^{i}\left(\bar{M}_{0, n}, T_{\bar{M}_{0, n}}(-\log (\mathcal{B}))=0\right.
$$

for any $i$. Now, let us consider the dual of the normal bundle $\mathcal{N}^{\vee}$. By [11, Lemma 4.2] the pull-back of $\mathcal{N}^{\vee}$ to $\bar{M}_{0, S_{1} \cup\left\{n_{1}+1\right\}} \times \bar{M}_{0, S_{2} \cup\left\{n_{2}+1\right\}}$ is identified with $p_{1}^{*} \psi_{n_{1}+1} \otimes p_{2}^{*} \psi_{n_{2}+1}$. Since $g=0$ we do not have the boundary divisor parametrizing irreducible nodal curves.

Recalling again the Kapranov's construction we have that the pull-back of $\mathcal{N}^{\vee}$ to $\bar{M}_{0, S_{1} \cup\left\{n_{1}+1\right\}} \times \bar{M}_{0, S_{2} \cup\left\{n_{2}+1\right\}}$ defines a birational morphism

$$
g:=f_{n_{1}+1} \times f_{n_{2}+1}: \bar{M}_{0, S_{1} \cup\left\{n_{1}+1\right\}} \times \bar{M}_{0, S_{2} \cup\left\{n_{2}+1\right\}} \rightarrow \mathbb{P}^{s_{1}-2} \times \mathbb{P}^{s_{2}-2},
$$

where $s_{i}=\left|S_{i}\right|$ for $i=1,2$. Then $\mathcal{N}=g^{*}(\mathcal{O}(-1,-1))$, where

$$
\mathcal{O}(-1,-1):=\mathcal{O}_{\mathbb{P}^{s_{1}}-2}(-1) \bigotimes \mathcal{O}_{\mathbb{P}^{s_{2}}-2}(-1) .
$$


By Künneth formula we have

$$
H^{i}\left(\mathbb{P}^{s_{1}-2} \times \mathbb{P}^{s_{2}-2}, \mathcal{O}(-1,-1)\right)=\bigoplus_{j+k=i} H^{j}\left(\mathbb{P}^{s_{1}-2}, \mathcal{O}_{\mathbb{P}^{s_{1}-2}}(-1)\right) \otimes H^{k}\left(\mathbb{P}^{s_{2}-2}, \mathcal{O}_{\mathbb{P}^{s_{2}-2}}(-1)\right)
$$

Therefore, we get $H^{i}\left(\mathbb{P}^{s_{1}-2} \times \mathbb{P}^{s_{2}-2}, \mathcal{O}(-1,-1)\right)=0$ for any $i$. Finally, applying to $\mathcal{N}^{\vee}$ the argument we used in the first part of the proof for $\omega_{\pi}(\Sigma)^{\vee}$ we get $H^{i}\left(\mathcal{B}^{v}, \mathcal{N}\right)=0$ for any $i$. Now, to conclude it is enough to apply Proposition 3.4.

As an immediate consequence of the proof of Theorem 4.1 we get the following result on the deformations of the pair $\left(\bar{M}_{0, n}, \partial \bar{M}_{0, n}\right)$, see [34, Section 3.3.3] for the general theory of deformations of the pair.

Corollary 4.2. Let $K$ be any field, $i \geq 0, n \geq 3$. Then,

$$
H^{i}\left(\bar{M}_{0, n}, T_{\bar{M}_{0, n}}(-\log (\mathcal{B}))\right)=0 .
$$

In particular, the pair $\left(\bar{M}_{0, n}, \partial \bar{M}_{0, n}\right)$ is rigid for any $n \geq 3$.

\section{Deformations of $\bar{M}_{1,2}$}

In this section, we prove that the moduli space $\bar{M}_{1,2}$ is not rigid. We work throughout over an algebraically closed field $K$ with $\operatorname{char}(K) \neq 2,3$.

\subsection{Preliminaries}

Choose $n$ and $a_{0}, \ldots, a_{n}$ positive integers. We will denote by $\mathbb{P}\left(a_{0}, \ldots, a_{n}\right)$ the associated weighted projective space, defined as the quotient scheme of the action of $K^{*}$ on $\mathbb{A}_{0}^{n+1}:=$ $\mathbb{A}^{n} \backslash\{0\}$ defined by

$$
\lambda \cdot\left(x_{0}, \ldots, x_{n}\right)=\left(\lambda^{a_{0}} x_{0}, \ldots, \lambda^{a_{n}} X_{n}\right) .
$$

The quotient $\mathbb{P}\left(a_{0}, \ldots, a_{n}\right)$ is a projective scheme with a quotient singularity of type $\frac{1}{a_{i}}\left(a_{0}, \ldots, \hat{a}_{i}, \ldots, a_{n}\right)$ at the $i$ th fundamental point.

Since we are in characteristic different from 2 and $3, \overline{\mathcal{M}}_{1,1}$ can be identified, using the Weierstrass form of the elliptic curve, with the weighted projective stack $\mathbb{P}(4,6)$ and $\overline{\mathcal{M}}_{1,2}$ is the stack quotient $\left[X /\left(K^{*} \times K^{*}\right)\right]$ where $X \subset \mathbb{A}_{0}^{3} \times \mathbb{A}_{0}^{2}$ is the closed subset defined by the equation

$$
X:=Z\left(z y^{2}-x^{3}-a x z^{2}-b z^{3}\right) \subset \mathbb{A}_{0}^{3} \times \mathbb{A}_{0}^{2}
$$


and the action is defined by

$$
\begin{array}{ccc}
\left(K^{*} \times K^{*}\right) \times X & \longrightarrow & X \\
((\lambda, \xi),(x, y, z, a, b)) & \longmapsto & \left(\xi \lambda^{2} X, \xi \lambda^{3} y, \xi z, \lambda^{4} a, \lambda^{6} b\right) .
\end{array}
$$

We denote by $E_{4}$ and $E_{6}$ the elliptic curves corresponding to the points $(1,0)$ and $(0,1)$ of $\overline{\mathcal{M}}_{1,1}$.

Lemma 5.1. Let $\mu_{4}$ act on $E_{4}$ via $\lambda \cdot(x, y, z)=\left(\lambda^{2} X, \lambda^{3} y, z\right)$; let $p_{1}=(0,1,0)$ and $p_{2}=$ $(0,0,1)$ be the fixed points. Then, $\lambda \in \mu_{4}$ acts on $H^{1}\left(E_{4}, T_{E_{4}}\left(-p_{1}\right)\right)$ by multiplication by $\lambda^{2}$ and on $T_{p_{1}} E_{4}$ and $T_{p_{2}} E_{4}$ by multiplication by $\lambda$.

Let $\mu_{6}$ act on $E_{6}$ via $\lambda \cdot(x, y, z)=\left(\lambda^{2} x, \lambda^{3} y, z\right)$; let $p_{1}=(0,1,0)$ be the fixed point, and $p_{2}=(0,0,1)$ the fixed point of the induced action of $\mu_{3}$. Then, $\mu_{6}$ acts on $H^{1}\left(E_{6}, T_{E_{6}}\left(-p_{1}\right)\right)$ by multiplication by $\lambda^{2}$ and on $T_{p_{1}} E_{6}$ by multiplication by $\lambda$. Finally, $\varepsilon \in \mu_{3}$ acts on $T_{p_{2}} E_{6}$ by multiplication by $\varepsilon$.

Proof. We consider the $E_{6}$ case. The function $\frac{x}{Y}$ is a local coordinate at the point $p_{1}$, hence the action of $\lambda$ on $\Omega_{p_{1}} E_{6}$ is given by multiplication by $\lambda^{-1}$, and therefore the action on the tangent space is given by $\lambda$. There is a canonical isomorphism $H^{1}\left(E_{6}, T_{E^{6}}\left(-p_{1}\right)\right) \rightarrow$ $H^{1}\left(E_{6}, T_{E^{6}}\right)$; the latter is $\mu_{6}$-equivariantly dual to $H^{0}\left(E_{6}, \Omega_{E_{6}}^{\otimes 2}\right)$ and by what we have just proven the action on one forms is given by $\lambda^{-2}$. The other cases are similar.

Let $(C, p)$ be a smooth or irreducible nodal elliptic curve, that is a point in $\overline{\mathcal{M}}_{1,1}$. Since the characteristic is different from 2 and 3 we can put $C$ in Weierstrass form, that is, there exists $(a, b) \in \mathbb{A}_{0}^{2}$ such that $(C, p)$ is isomorphic to $\left(C_{a, b},[0: 1: 0]\right)$, where

$$
C_{a, b}=Z\left(z y^{2}-x^{3}-a x z^{2}-b z^{3}\right) \subset \mathbb{P}^{2} .
$$

This representation is called Weierstrass representation of the elliptic curve. Moreover, the set of isomorphisms between $\left(C_{a, b},[0: 1: 0]\right)$ and $\left(C_{\bar{a}, \bar{b}},[0: 1: 0]\right)$ can be identified with $\left\{\lambda \in K \mid(\bar{a}, \bar{b})=\left(\lambda^{4} a, \lambda^{6} b\right)\right\}$ by associating to each such $\lambda$ the map $\phi_{\lambda}(x, y, z)=$ $\left(\lambda^{2} x, \lambda^{3} y, z\right)$. Using the representation (5.2) we have

$$
E_{4}:=\left\{y^{2} z=x^{3}+x z^{2}\right\} \subset \mathbb{P}^{2}, \quad E_{6}:=\left\{y^{2} z=x^{3}+z^{3}\right\} \subset \mathbb{P}^{2} .
$$

The rational Picard group of $\bar{M}_{1,2}$ is freely generated by the two boundary divisors [28]: the divisors $\Delta_{\text {irr }}$ parametrizing irreducible nodal curves, and $\Delta_{1}$ parametrizing elliptic 
tails. These divisors are both smooth, rational curves. The boundary divisor $\Delta_{1}$ has negative self-intersection. In the following, we give an explicit description of the contraction of $\Delta_{1}$.

\section{$5.2 \bar{M}_{1,2}$ as toric variety}

Assume $n$ is an integer prime to the characteristic of $K$. We denote by $\frac{1}{n}\left(a_{1}, a_{2}\right)$ any surface singularity which is étale locally isomorphic to $\operatorname{Spec}\left(K\left[t_{1}, t_{2}\right]^{\mu_{n}}\right)$, where $\mu_{n}$ acts by $\lambda \cdot\left(x_{1}, X_{2}\right)=\left(\lambda^{a_{1}} X_{1}, \lambda^{a_{2}} X_{2}\right)$.

Proposition 5.2. The moduli space $\bar{M}_{1,2}$ is a surface with four singular points. Two singular points lie in $M_{1,2}$, and are

- a singularity of type $\frac{1}{2}(1,1)$ at the image of $(0,0,1,1,0) \in X$, representing an elliptic curve of Weierstrass representation $E_{4}$ with marked points $[0: 1: 0]$ and $[0: 0: 1]$;

- a singularity of type $\frac{1}{3}(1,2)$ at the image of $(0,1,1,0,1) \in X$, representing an elliptic curve of Weierstrass representation $E_{6}$ with marked points [0 $\left.: 1: 0\right]$ and $[0: 1: 1]$.

The remaining two singular points lie on the boundary divisor $\Delta_{1}$, and are

- a singularity of type $\frac{1}{3}(1,1)$ at the image of $(0,1,0,1,0) \in X$, representing a reducible curve whose irreducible components are an elliptic curve of type $E_{6}$ and a smooth rational curve connected by a node;

- a singularity of type $\frac{1}{2}(1,1)$ at the image of $(0,1,0,0,1) \in X$, representing a reducible curve whose irreducible components are an elliptic curve of type $E_{4}$ and a smooth rational curve connected by a node.

Proof. On $X, z=0 \Rightarrow x=0 \Rightarrow y \neq 0$. So $X$ is covered by the charts $\{z \neq 0\}$ and $\{y \neq 0\}$. Consider first the chart $\{z \neq 0\}$. Then we may take $\xi=1$. On this chart $X$ is given by $\left\{y^{2}=x^{3}+a x+b\right\}$ so $b=y^{2}-x^{3}-a x$. We can take $(x, y, a)$ as coordinates, and the action of $K^{*} \times K^{*}$ is given by $(\lambda, x, y, a) \mapsto\left(\lambda^{2} x, \lambda^{3} y, \lambda^{4} a\right)$. The point $(0,0,0)$ is stabilized by $K^{*} \times K^{*}$, so does not produce any singularity. Since $(2,3)=(3,4)=1$, the points $(x, y, a)$ such that $x y \neq 0$ or $y a \neq 0$ have trivial stabilizer. 
If $y=0$ the action is given by $(\lambda, x, a) \mapsto\left(\lambda^{2} x, \lambda^{4} a\right)$. If $x=0$ then $a \neq 0$, the stabilizer is $\mu_{4}$. So on the chart $a \neq 0$ we have a singularity of type $\frac{1}{2}(1,1)$. Note that $x=y=0$ implies $b=0$. The singular point corresponds to a smooth elliptic curve of Weierstrass form $E_{4}$ and whose second marked point is [0:0:1]. If $x \neq 0$, then the stabilizer is $\mu_{2}$ and on this chart we find points of type $\frac{1}{2}(1,0)$ and these are smooth points. If $y \neq 0$, then $\xi \lambda^{3}=\lambda^{3}=1$ and we get a singularity of type $\frac{1}{3}(1,2)$ at the point $a=x=0$.

Consider now the locus $\{z=0\}$. We can take $y=1$. Then $\xi \lambda^{3}=1$ and $X$ is given by $\left\{z=x^{3}+a x z^{2}+b z^{3}\right\}$. We are interested in a neighborhood of $x=z=0$. Let $f(x, z, a, b)=z-x^{3}-a x z^{2}-b z^{3}$ be the polynomial defining $X$. Since $\frac{\partial f}{\partial z} \mid z=0=0$ we can choose $(x, a, b)$ as local coordinates. The action is given by $(\lambda, \xi, x, a, b) \mapsto\left(\xi \lambda^{2} x, \lambda^{4} a, \lambda^{6} b\right)$. If $x \neq 0$, the stabilizer is trivial. If $x=0$ and $a b \neq 0$ the stabilizer is $\mu_{2}$ and does not produce any singularity. If $a=0, b \neq 0$, then $\lambda^{6}=1$. This yields a singular point of type $\frac{1}{3}(1,1)$. If $a \neq 0, b=0$, then $\lambda^{4}=1$ and we get a singular point of type $\frac{1}{2}(1,1)$.

\subsubsection{Weighted blow-ups}

The weighted blow-up of $\mathbb{A}^{2}$ with weights $\left(a_{1}, a_{2}\right)$ where $\operatorname{gcd}\left(a_{1}, a_{2}\right)=1$ is a projective birational morphism $f: X \rightarrow \mathbb{A}^{2}$ such that $X$ is covered by two affine charts $U_{1} \cong \mathbb{A}^{2} / \mu_{a_{1}}$ where the action is given by $\lambda \cdot\left(y_{1}, y_{2}\right)=\left(\lambda y_{1}, \lambda^{-a_{2}} y_{2}\right)$, and $U_{2} \cong \mathbb{A}^{2} / \mu_{a_{2}}$ where $\lambda \cdot\left(y_{1}, y_{2}\right)=\left(\lambda^{-a_{1}} y_{1}, \lambda y_{2}\right)$. The exceptional divisor $E$ is given in $U_{i}$ by $\left\{y_{i}=0\right\}$. Therefore, $X$ has two finite quotient singularities of type

$$
\frac{1}{a_{1}}\left(1,-a_{2}\right) \text { and } \frac{1}{a_{2}}\left(-a_{1}, 1\right)
$$

on the exceptional divisor $E=\mathbb{P}\left(a_{1}, a_{2}\right) \cong \mathbb{P}^{1}$ corresponding to the points [1:0], [0:1] of $E$, respectively. By $[13$, Section 3$] X$ can be constructed as a follows. Let $C=\mathbb{R}_{+} e_{1}+\mathbb{R}_{+} e_{2}$ be the cone in $\mathbb{R}^{2}$ whose rays are $e_{1}=(1,0), e_{2}=(0,1)$. We divide the cone $C$, in two cones $C_{1}$ and $C_{2}$, by adding the one-dimensional cone $\mathbb{R}_{+} e_{3}$, where $e_{3}=\left(a_{1}, a_{2}\right)$. Let $\Sigma_{a_{1}, a_{2}}$ be the fan consisting of all the faces of $C_{1}, C_{2}$. Then, $X$ is the toric variety determined by the lattice $N=\mathbb{Z} e_{1}+\mathbb{Z} e_{2}+\mathbb{Z} e_{3}$ and the fan $\Sigma_{a_{1}, a_{2}}$.

Theorem 5.3. The moduli space $\bar{M}_{1,2}$ is isomorphic to the weighted blow-up with weights $(2,3)$ of the weighted projective plane $\mathbb{P}(1,2,3)$ in its smooth point $[1: 0: 0]$. 
That is $\bar{M}_{1,2}$ is the toric variety associated with toric fan $\Sigma$ in the following picture:

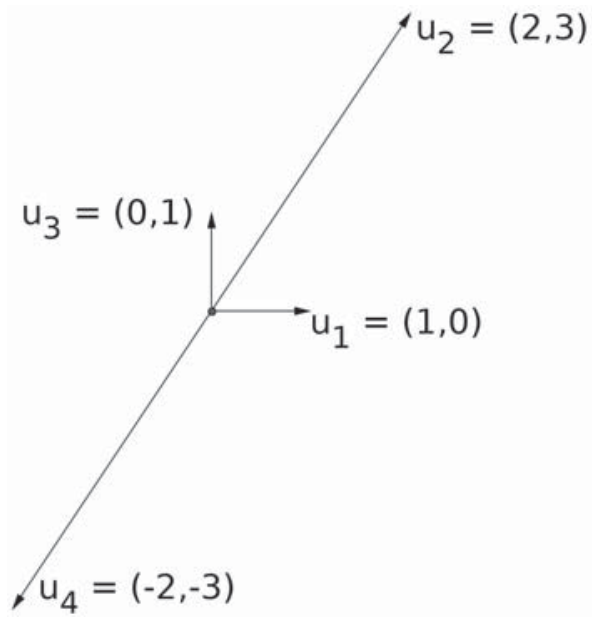

Proof. Recall the description of $\bar{M}_{1,2}$ given via (5.1). On the chart $\mathcal{U}_{z}:=\{z \neq 0\}$, we define a morphism

$$
\begin{aligned}
& f_{\mathcal{U}_{z}}: \quad \mathcal{U}_{z} \quad \longrightarrow \quad \mathbb{P}(1,2,3) \\
& (x, y, z, a, b) \longmapsto\left(x, a z^{2}, b z^{3}\right) .
\end{aligned}
$$

The action of $K^{*} \times K^{*}$ on this triple is given by $\left(\xi \lambda^{2}, \xi^{2} \lambda^{4}, \xi^{3} \lambda^{6}\right)$, and $f_{\mathcal{U}_{z}}$ is indeed a welldefined morphism to $\mathbb{P}(1,2,3)$. The morphism $f_{\mathcal{U}_{z}}$ maps the two singular points in $M_{1,2}$ we found in Proposition 5.2 in the points $[0: 1: 0],[0: 0: 1] \in \mathbb{P}(1,2,3)$, which are the only singularities of the weighted projective plane and of the same type of the singularities on $M_{1,2}$.

On $\mathcal{U}_{y}:=\{y \neq 0\}$ the equation of $\bar{M}_{1,2}$ is $z=x^{3}+a x z^{2}+b z^{3}$. So, as explained in the proof of Proposition $5.2 x$ is a local parameter near $z=0$. We can consider the morphism

$$
f_{\mathcal{U}_{Y}}(x, y, z, a, b)=\left(1, a\left(\frac{x^{2}+a z^{2}}{1-b z^{2}}\right)^{2}, b\left(\frac{x^{2}+a z^{2}}{1-b z^{2}}\right)^{3}\right)
$$

From this formulation, it is clear that $f_{\mathcal{U}_{Y}}$ is defined even on the locus $\{x=0\}$ and the divisor $\Delta_{1}=\{x=z=0\}$ is contracted in the smooth point [1:0:0] of $\mathbb{P}(1,2,3)$. On $\mathcal{U}_{z} \cap \mathcal{U}_{Y}$ we have $\frac{z}{x}=\frac{x^{2}+a z^{2}}{1-b z^{2}}$ and $f_{\mathcal{U}_{z}}=f_{\mathcal{U}_{Y}}$, so $f_{\mathcal{U}_{z}}, f_{\mathcal{U}_{Y}}$ glue to a morphism

$$
f: \bar{M}_{1,2} \rightarrow \mathbb{P}(1,2,3)
$$


Since, by [28] the Picard number of $\bar{M}_{1,2}$ is 2, $\Delta_{1}$ must be the only divisor contracted by $f$. Let $\xi \in \mathbb{P}(1,2,3)$ be a point $\xi \neq[1: 0: 0]$. Since by $(5.2) z=0$ forces $x=0$ we have that $f^{-1}(\xi)$ is contained in the subset $\mathcal{U}_{z}$ where $z \neq 0$, and we may consider the expression for $f_{\mathcal{U}_{z}}$ in (5.4). By (5.2) a point in the fiber $f_{\mathcal{U}_{z}}^{-1}(\xi)$ is determined, up to a sign, by the variable $y$. Therefore, in the quotient (5.1) such a point in uniquely determined. This means that $f_{\mid \bar{M}_{1,2} \backslash \Delta_{1}}: \bar{M}_{1,2} \backslash \Delta_{1} \rightarrow \mathbb{P}(1,2,3) \backslash\{[1: 0: 0]\}$ is an isomorphism. We conclude that $f: \bar{M}_{1,2} \rightarrow \mathbb{P}(1,2,3)$ is indeed a birational morphism with exceptional locus $\Delta_{1}$. Then, by [32, Proposition 6.2.6] $f$ is a weighted blow-up of $\mathbb{P}(1,2,3)$ in $[1: 0: 0]$ and $\Delta_{1}$ is the corresponding exceptional divisor. By Proposition 5.2 we know that $\bar{M}_{1,2}$ has two singularities of type $\frac{1}{2}(1,1), \frac{1}{3}(1,1)$ on $\Delta_{1}$. By Proposition 5.2 we know that the singularities $\frac{1}{2}(1,1), \frac{1}{3}(1,1) \in \Delta_{1}$ corresponds to $b=0, a=0$, respectively. By (5.3) this forces the weights of the blow-up to be $(2,3)$.

With this description of $\bar{M}_{1,2}$ we can easily compute its automorphism group.

Proposition 5.4. Let $K$ be an algebraically closed field with $\operatorname{char}(K) \neq 2,3$. The automorphism group of $\bar{M}_{1,2}$ is isomorphic to $\left(K^{*}\right)^{2}$.

Proof. Let $\pi: \bar{M}_{1,2} \rightarrow \bar{M}_{1,1}$ be a forgetful morphism, and $F_{i}=\pi^{-1}\left(\left[E_{i}\right]\right)$ for $i=4,6$. Let $f: \bar{M}_{1,2} \rightarrow \mathbb{P}(1,2,3)=\operatorname{Proj}\left(K\left[x_{0}, x_{1}, x_{2}\right]\right)$ be the weighted blow-up described in Theorem 5.3 , where the grading on $K\left[x_{0}, x_{1}, x_{2}\right]$ is given by $\operatorname{deg}\left(x_{i}\right)=i+1$. Any $\phi \in \operatorname{Aut}\left(\bar{M}_{1,2}\right)$ must stabilize $\Delta_{1}, F_{4}$, and $F_{6}$.

Therefore, we have an injective morphism $\chi: \operatorname{Aut}\left(\bar{M}_{1,2}\right) \rightarrow G \subset \operatorname{Aut}(\mathbb{P}(1,2,3))$ where $G$ consists of automorphisms of $\mathbb{P}(1,2,3)$ fixing $[1: 0: 0]$ and stabilizing $f\left(F_{4}\right)=$ $\left\{x_{2}=0\right\}$ and $f\left(F_{6}\right)=\left\{x_{1}=0\right\}$. To conclude that $G \cong\left(K^{*}\right)^{2}$, it is enough to recall that the automorphisms of $\mathbb{P}(1,2,3)$ are the automorphisms of the graded $K$-algebra $K\left[x_{0}, X_{1}, x_{2}\right]$. By Theorem $5.3 \bar{M}_{1,2}$ is toric. To conclude it is enough to observe that the inclusion $i:\left(K^{*}\right)^{2} \hookrightarrow \operatorname{Aut}\left(\bar{M}_{1,2}\right)$ is a section of $\chi$.

For the rest of this section, $K$ will be an algebraically closed field of characteristic zero. The cohomology of a toric invariant divisor $D$ in a toric variety $X_{\Sigma}$ can be computed in terms of combinatorial data encoded in the dual lattice of the variety, and in a polytope $P_{D}$ associated with $D$, see $[4$, Chapters 4,9$]$. This leads us to the following result.

Proposition 5.5. Let $X_{\Sigma}$ we the weighted blow-up with weights $(2,3)$ of $\mathbb{P}(1,2,3)$ in [1:0:0]. Then, $h^{0}\left(X_{\Sigma}, T_{X_{\Sigma}}\right)=2$ and $h^{i}\left(X_{\Sigma}, T_{X_{\Sigma}}\right)=0$ for $i=1,2$. 
Proof. Let $D_{\rho}$ be the toric divisor corresponding to the ray $u_{\rho}$ in the fan $\Sigma$ of Theorem 5.3, and let $\mathcal{O}_{X_{\Sigma}}\left(D_{\rho}\right)$ be the associated sheaf. The cohomology of $\mathcal{O}_{X_{\Sigma}}\left(D_{\rho}\right)$ can be computed in terms of lattice objects. Indeed, by [4, Proposition 4.3.3] we have $h^{0}\left(X_{\Sigma}, \mathcal{O}_{X_{\Sigma}}\left(D_{\rho}\right)\right)=1$ for $\rho=1,2,3,4$, while by [4, Proposition 9.1.6] we get $h^{1}\left(X_{\Sigma}, \mathcal{O}_{X_{\Sigma}}\left(D_{\rho}\right)\right)=h^{2}\left(X_{\Sigma}, \mathcal{O}_{X_{\Sigma}}\left(D_{\rho}\right)\right)=0$ for $\rho=1,2,3,4$.

Since the class group of $X_{\Sigma}$ if free of rank 2, by [4, Theorem 8.1.6] we have the following exact sequence

$$
0 \mapsto \Omega_{X_{\Sigma}} \rightarrow \bigoplus_{\rho=1}^{4} \mathcal{O}_{X_{\Sigma}}\left(-D_{\rho}\right) \rightarrow \mathcal{O}_{X_{\Sigma}}^{\oplus 2} \mapsto 0
$$

and dualizing we get

$$
0 \mapsto \mathcal{O}_{X_{\Sigma}}^{\oplus 2} \rightarrow \bigoplus_{\rho=1}^{4} \mathcal{O}_{X_{\Sigma}}\left(D_{\rho}\right) \rightarrow T_{X_{\Sigma}} \mapsto 0
$$

To conclude, it is enough to take cohomology and to use the results on the cohomology of the toric divisors in the first part of the proof.

Remark 5.6. By [4, Proposition 6.4.4] we can compute the intersection matrix of the $D_{\rho}$ 's. This is given by

$$
\left(D_{i} \cdot D_{j}\right)=\left(\begin{array}{cccc}
0 & \frac{1}{3} & 0 & \frac{1}{3} \\
\frac{1}{3} & -\frac{1}{6} & \frac{1}{2} & 0 \\
0 & \frac{1}{2} & 0 & \frac{1}{2} \\
\frac{1}{3} & 0 & \frac{1}{2} & \frac{1}{6}
\end{array}\right)
$$

Therefore $D_{\rho}$ for $\rho=1,3,4$ is nef, and the vanishing of $H^{i}\left(X_{\Sigma}, \mathcal{O}_{X_{\Sigma}}\left(D_{\rho}\right)\right)$ for $i=1,2$, and $\rho=1,3,4$ can be deduced also from Demazure's vanishing theorem [4, Theorem 9.2.3].

Remark 5.7. By Proposition 5.4 Aut $\left(\bar{M}_{1,2}\right) \cong\left(K^{*}\right)^{2}$, and indeed by Proposition 5.5 we have that $T_{[I d]} \operatorname{Aut}\left(\bar{M}_{1,2}\right) \cong H^{0}\left(\bar{M}_{1,2}, T_{\bar{M}_{1,2}}\right)$ is of dimension 2 .

Now, we are ready to compute the Kuranishi family of $\bar{M}_{1,2}$ by computing the deformations of the singularities of $\bar{M}_{1,2}$ described in Proposition 5.2. See [1] for a survey on deformations of singularities. 
Theorem 5.8. The coarse moduli space $\bar{M}_{1,2}$ does not have locally trivial deformations, while its family of first-order infinitesimal deformations is non-singular of dimension 6 and the general deformation is smooth.

Proof. The first-order infinitesimal deformations of the surface $\bar{M}_{1,2}$ are parametrized by the group $\operatorname{Ext}^{1}\left(\Omega_{\bar{M}_{1,2}}, \mathcal{O}_{\bar{M}_{1,2}}\right)$. The sheaf $\mathcal{E} x t^{1}\left(\Omega_{\bar{M}_{1,2}} \mathcal{O}_{\bar{M}_{1,2}}\right)$ is supported on the singularities and since $\bar{M}_{1,2}$ has isolated singularities $\mathcal{E} x t^{1}\left(\Omega_{\bar{M}_{1,2}}, \mathcal{O}_{\bar{M}_{1,2}}\right)$ can be computed separately for each singular point.

Let us consider the singular point $p \in \bar{M}_{1,2}$ of type $\frac{1}{3}(1,2)$. Then, étale locally, in a neighborhood of $p$ the surface $\bar{M}_{1,2}$ is isomorphic to $\mathbb{A}^{2} / \mu_{3}$ where the action is given by

$$
\begin{aligned}
& \mu_{3} \times \mathbb{A}^{2} \longrightarrow \mathbb{A}^{2} \\
& \left(\epsilon, X_{1}, X_{2}\right) \longmapsto\left(\epsilon X_{1}, \epsilon^{2} X_{2}\right) \text {. }
\end{aligned}
$$

The invariant polynomials with respect to this action are clearly $x_{1}^{3}, x_{2}^{3}, x_{1} x_{2}$. Therefore, étale locally, in a neighborhood of $p$ the surface $\bar{M}_{1,2}$ is isomorphic to an étale neighborhood of the singularity

$$
S=\left\{f(x, y, z)=z^{3}-x y=0\right\} \subset \mathbb{A}^{3} .
$$

Let $R=K[x, y, z] /\left(z^{3}-x y\right)$, and let us consider the free resolution

$$
0 \mapsto R \stackrel{\psi_{J}}{\longrightarrow} R^{\oplus 3} \longrightarrow \Omega_{R} \mapsto 0
$$

of $\Omega_{R}$, where $\psi_{J}$ is the matrix of the partial derivatives of $f=z^{3}-x y$. Therefore, we get

$$
\operatorname{Ext}^{1}\left(\Omega_{R}, R\right) \cong R / \operatorname{Im}\left(\psi_{J}^{t}\right) \cong K[x, y, z] /\left(z^{3}-x y,-y,-x, 3 z^{2}\right) \cong K[z] /\left(z^{2}\right), \operatorname{Ext}^{2}\left(\Omega_{R}, R\right)=0 .
$$

The same argument for the two singularities of type $\frac{1}{2}(1,1)$ shows that in these cases we have

$$
\operatorname{Ext}^{1}\left(\Omega_{R}, R\right) \cong K[x, y, z] /\left(z^{2}-x y,-y,-X, 2 z\right) \cong K, \operatorname{Ext}^{2}\left(\Omega_{R}, R\right)=0 .
$$

Now, let us consider the singularity of type $\frac{1}{3}(1,1)$. In this case, the action is given by

$$
\begin{array}{ccc}
\mu_{3} \times \mathbb{A}^{2} & \longrightarrow & \mathbb{A}^{2} \\
\left(\epsilon, X_{1}, X_{2}\right) & \longmapsto & \left(\epsilon X_{1}, \epsilon X_{2}\right) .
\end{array}
$$


The invariants are clearly $x_{1}^{3}, x_{1}^{2} x_{2}, x_{1} x_{2}^{2}, x_{2}^{3}$. Then étale locally, in a neighborhood of this singularity the surface $\bar{M}_{1,2}$ is isomorphic to an étale neighborhood of the vertex of a cone in $\mathbb{A}^{4}$ over a twisted cubic in $\mathbb{P}^{3}$. The coordinate ring of such a cone is $R=$ $K[x, y, z, w] /\left(f_{1}, f_{2}, f_{3}\right)$, where $f_{1}=x w-y z, f_{2}=y^{2}-x z$ and $f_{3}=z^{2}-y w$. We get a free resolution of the module of differentials $\Omega_{R}$ as follows:

$$
R^{\oplus 3} \stackrel{\psi}{\longrightarrow} R^{\oplus 2} \stackrel{\psi_{S}}{\longrightarrow} R^{\oplus 3} \stackrel{\psi_{J}}{\longrightarrow} R^{\oplus 4} \longrightarrow \Omega_{R} \rightarrow 0,
$$

where

$$
\psi=\left(\begin{array}{ccc}
x & z & y \\
-y & -w & -z
\end{array}\right), \quad \psi_{S}=\left(\begin{array}{cc}
z & y \\
w & z \\
y & x
\end{array}\right), \quad \psi_{J}=\left(\begin{array}{ccc}
w & -z & 0 \\
-z & 2 y & -w \\
-y & -x & 2 z \\
x & 0 & -y
\end{array}\right)
$$

Note that $\psi_{S}$ and $\psi_{J}$ are the syzygy matrix and the Jacobian matrix of the $f_{i}^{\prime}$ s, respectively. We may compute $\operatorname{Ext}^{1}\left(\Omega_{R}, R\right) \cong \operatorname{ker}\left(\psi_{S}^{t}\right) / \operatorname{Im}\left(\psi_{J}^{t}\right)$. Now, $\operatorname{ker}\left(\psi_{S}^{t}\right)$ has the following six generator: $(-W, z, 0),(z,-y, 0),(0, y,-W),(y, 0,-z),(0,-x, z),(-x, 0, y)$, and $\operatorname{Im}\left(\psi_{J}^{t}\right)$ is generated by $(w,-z, 0),(-z, 2 y,-w),(-y,-x, 2 z),(x, 0,-y)$. Furthermore, $\operatorname{ker}\left(\psi^{t}\right)$ is generated by $(W, z),(z, y),(y, x)$, which are generators for $\operatorname{Im}\left(\psi_{S}^{t}\right)$ as well. Therefore,

$$
\operatorname{dim}_{K} \operatorname{Ext}^{1}\left(\Omega_{R}, R\right)=2, \operatorname{Ext}^{2}\left(\Omega_{R}, R\right)=0
$$

This last fact together with (5.5) and (5.6) implies that

$$
h^{0}\left(\bar{M}_{1,2}, \mathcal{E} x t^{1}\left(\Omega_{\bar{M}_{1,2}}, \mathcal{O}_{\bar{M}_{1,2}}\right)\right)=2+1+1+2=6 .
$$

Now, by Theorem 5.3 the surface $\bar{M}_{1,2}$ is a weighted blow-up of $\mathbb{P}(1,2,3)$ in its smooth point $[1: 0: 0]$ that is the variety $X_{\Sigma}$ in Proposition 5.5. Therefore, Proposition 5.5 yields $h^{0}\left(\bar{M}_{1,2}, T_{\bar{M}_{1,2}}\right)=2$ and

$$
H^{i}\left(\bar{M}_{1,2}, T_{\bar{M}_{1,2}}\right)=0 \quad \text { for } \quad i=1,2 .
$$

So the sequence

$$
H^{1}\left(\bar{M}_{1,2}, T_{\bar{M}_{1,2}}\right) \rightarrow \operatorname{Ext}^{1}\left(\Omega_{\bar{M}_{1,2}}, \mathcal{O}_{\bar{M}_{1,2}}\right) \rightarrow H^{0}\left(\bar{M}_{1,2}, \mathcal{E} X t^{1}\left(\Omega_{\bar{M}_{1,2}} \mathcal{O}_{\bar{M}_{1,2}}\right)\right) \rightarrow H^{2}\left(\bar{M}_{1,2}, T_{\bar{M}_{1,2}}\right)
$$

yields $\operatorname{Ext}^{1}\left(\Omega_{\bar{M}_{1,2}} \mathcal{O}_{\bar{M}_{1,2}}\right) \cong H^{0}\left(\bar{M}_{1,2}, \mathcal{E x t}^{1}\left(\Omega_{\bar{M}_{1,2}} \mathcal{O}_{\bar{M}_{1,2}}\right)\right)$. Finally, to compute the dimension of the obstruction space $\operatorname{Ext}^{2}\left(\Omega_{\bar{M}_{1,2}} \mathcal{O}_{\bar{M}_{1,2}}\right)$ we use the local-to-global Ext spectral 
sequence

$$
H^{i}\left(\bar{M}_{1,2}, \mathcal{E} \mathrm{xt}^{j}\left(\Omega_{\bar{M}_{1,2}}, \mathcal{O}_{\bar{M}_{1,2}}\right)\right) \Rightarrow \operatorname{Ext}^{i+j}\left(\Omega_{\bar{M}_{1,2}}, \mathcal{O}_{\bar{M}_{1,2}}\right)
$$

Clearly, $H^{1}\left(\bar{M}_{1,2}, \mathcal{E} \mathrm{xt}^{1}\left(\Omega_{\bar{M}_{1,2}}, \mathcal{O}_{\bar{M}_{1,2}}\right)\right)=0$ because $\mathcal{E} \mathrm{xt}^{1}\left(\Omega_{\bar{M}_{1,2}}, \mathcal{O}_{\bar{M}_{1,2}}\right)$ is supported on a zero-dimensional scheme. Furthermore, by (5.8) we have $H^{2}\left(\bar{M}_{1,2}, \mathcal{E x t}^{0}\left(\Omega_{\bar{M}_{1,2}}, \mathcal{O}_{\bar{M}_{1,2}}\right)\right)=$ $H^{2}\left(\bar{M}_{1,2}, T_{\bar{M}_{1,2}}\right)=0$. Finally (5.5)-(5.7) yield $H^{0}\left(\bar{M}_{1,2}, \mathcal{E} \mathrm{xt}^{2}\left(\Omega_{\bar{M}_{1,2}}, \mathcal{O}_{\bar{M}_{1,2}}\right)\right)=0$ as well.

\section{On the Deformations of the Coarse Moduli Space $\bar{M}_{g, n}$}

In this section, we will study the infinitesimal deformations of the coarse moduli space $\bar{M}_{g, n}$, which is a projective normal scheme with finite quotient singularities. If $g=0$ we have $\bar{M}_{0, n} \cong \overline{\mathcal{M}}_{0, n}$, by [11, Theorem 2.1] in characteristic zero, and Theorem 4.1 in positive characteristic we know that $\bar{M}_{0, n}$ is rigid, hence we restrict to the case $g \geq 1$.

\subsection{Deformation theory for varieties with quotient singularities}

Let $X$ be a variety over a field $K, A$ an Artinian $K$-algebra with residue field $K$. A deformation $X_{A}$ of $X$ over $\operatorname{Spec}(A)$ is called trivial if it is isomorphic to $X \times_{K} \operatorname{Spec}(A)$; it is locally trivial if there is an open cover of $X$ by open affines $U$ such that the induced deformation $U_{A}$ is trivial.

We recall some well-known facts about infinitesimal deformations of normal varieties. By [14] the tangent and obstruction spaces to deformations of $X$ are given by $\operatorname{Ext}^{1}\left(L_{X}, \mathcal{O}_{X}\right)$ and $\operatorname{Ext}^{2}\left(L_{X}, \mathcal{O}_{X}\right)$ where $L_{X}$ is the cotangent complex; when $X$ is a normal variety, these spaces are actually $\operatorname{Ext}^{1}\left(\Omega_{X}, \mathcal{O}_{X}\right)$ and $\operatorname{Ext}^{2}\left(\Omega_{X}, \mathcal{O}_{X}\right)$, respectively. Locally trivial infinitesimal deformations have as tangent and obstruction spaces $H^{1}\left(X, T_{X}\right)$ and $H^{2}\left(X, T_{X}\right)$, respectively.

Remark 6.1. By the exact sequence

$$
0 \mapsto H^{1}\left(X, T_{X}\right) \rightarrow \operatorname{Ext}^{1}\left(\Omega_{X}, \mathcal{O}_{X}\right) \rightarrow H^{0}\left(X, \mathcal{E x t}^{1}\left(\Omega_{X}, \mathcal{O}_{X}\right)\right) \rightarrow H^{2}\left(X, T_{X}\right) \rightarrow \operatorname{Ext}^{2}\left(\Omega_{X}, \mathcal{O}_{X}\right)
$$

induced by the local-to-global spectral sequence for Ext, if $H^{0}\left(X, \mathcal{E x t}^{1}\left(\Omega_{X}, \mathcal{O}_{X}\right)\right)=0$ then all deformations are locally trivial, while if $H^{1}\left(X, T_{X}\right)=0$ then all locally trivial deformations are trivial.

Remark 6.2. The sheaf $\mathcal{E} \operatorname{xt}^{1}\left(\Omega_{X}, \mathcal{O}_{X}\right)$ is supported on the singular locus of $X$; if $X$ has quotient singularities, then by [6, Lemmas 2.4,2.5] the sheaf of cohomology with supports $\mathcal{H}_{Z}^{0}\left(\mathcal{E x t}^{1}\left(\Omega_{X}, \mathcal{O}_{X}\right)\right)=0$ if $Z \subset X$ is a closed subset of codimension $\geq 3$. 


\subsection{Locally trivial deformations of $\bar{M}_{g, n}$}

Let $\mathcal{X}$ be a smooth Deligne-Mumford stack, and let $\pi: \mathcal{X} \rightarrow X$ be the structure morphism on its coarse module space. The stack $\mathcal{X}$ is called canonical if the locus where $\pi$ is not an isomorphism has dimension $\leq \operatorname{dim}(\mathcal{X})-2$ [8, Definition 4.4]. Every variety with quotient singularities is the coarse moduli space of a canonical smooth Deligne-Mumford stack, unique up to unique isomorphism [8, Remark 4.9].

Lemma 6.3. Let $X$ be a variety with finite quotient singularities and let $\mathcal{X}$ be its canonical stack. Then, $H^{i}\left(X, T_{X}\right) \cong H^{i}\left(\mathcal{X}, T_{\mathcal{X}}\right)$ for any $i \geq 0$. In particular, if $\mathcal{X}$ is rigid then $X$ does not have locally trivial deformations.

Proof. Let $Z=\operatorname{Sing}(X)$ and $U=X \backslash Z$. Since $\mathcal{X}$ is the canonical stack of $X$, we may embed $U$ in $\mathcal{X}$ as well. We get the following commutative diagram:

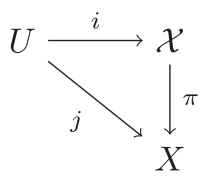

and $\pi_{*} i_{*} T_{U}=j_{*} T_{U}$. Furthermore, we have the two following exact sequences:

$$
\begin{aligned}
0 & \mapsto \mathcal{H}_{Z}^{0}\left(X, T_{X}\right) \rightarrow T_{X} \rightarrow j_{*} T_{X \mid U} \rightarrow \mathcal{H}_{Z}^{1}\left(X, T_{X}\right), \\
0 & \mapsto \mathcal{H}_{W}^{0}\left(\mathcal{X}, T_{\mathcal{X}}\right) \rightarrow T_{\mathcal{X}} \rightarrow i_{*} T_{\mathcal{X} \mid U} \rightarrow \mathcal{H}_{W}^{1}\left(\mathcal{X}, T_{\mathcal{X}}\right),
\end{aligned}
$$

where $W=\pi^{-1}(Z)$ with the reduced substack structure, and $\mathcal{H}_{Z}^{i}$ is the sheaf of cohomology with supports (see [10]). Furthermore, since $\operatorname{codim}_{X}(Z)=\operatorname{codim}_{\mathcal{X}}(W) \geq 2$ we get $\mathcal{H}_{Z}^{i}\left(X, T_{X}\right)=\mathcal{H}_{W}^{i}\left(\mathcal{X}, T_{\mathcal{X}}\right)=0$ for $i<2$. This yields $\pi_{*} T_{\mathcal{X}} \cong \pi_{*} i_{*} T_{U} \cong j_{*} T_{U} \cong T_{X}$. Finally, since $R^{i} \pi_{*} T_{\mathcal{X}}=0$ for $i \geq 1$, because $\pi$ is finite, we conclude by Leray's spectral sequence.

Lemma 6.4. Let $c: \overline{\mathcal{M}}_{g, n} \rightarrow \overline{\mathcal{M}}_{g, n}^{\text {can }}$ be the structure map on the canonical stack. If $g+n \geq 4$, it is an isomorphism outside the boundary divisor $\Delta_{1}$ of elliptic tails, that is, the image of $\overline{\mathcal{M}}_{1,1} \times \overline{\mathcal{M}}_{g-1, n+1}$ via the gluing map.

Proof. By [3, Corollary 1] if $g+n \geq 4$ any component of the locus parametrizing curves in $M_{g, n}$ with a non-trivial automorphism has codimension at least 2.

Let us consider a general point $\left[C, X_{1}, \ldots, X_{n}\right]$ of the boundary divisor $\Delta_{\text {irr }}$ parametrizing irreducible nodal curves. The normalization $v: \widetilde{C} \rightarrow C$ of $C$ is a 
smooth curve of genus $g-1$. Under our numerical hypothesis $\left[\widetilde{C}, v^{-1}\left(x_{1}\right), \ldots, v^{-1}\left(x_{n}\right)\right]$ is automorphism-free. Therefore, $\left[C, X_{1}, \ldots, x_{n}\right]$ does not have non-trivial automorphisms as well.

The remaining boundary divisors parametrize reducible curves $\left[C_{1} \cup\right.$ $\left.C_{2}, X_{1}, \ldots, x_{n}\right]$ where $C_{1}, C_{2}$ are smooth curves of genus $g_{1}, g_{2}$ with $n_{1}, n_{2}$ marked points, respectively, intersecting just in one node $p=C_{1} \cap C_{2}$, and such that $g_{1}+g_{2}=g$ and $n_{1}+n_{2}=n$. Recalling that a general stable curve of the form $\left[C_{i}, x_{i_{1}}, \ldots, x_{i_{1}}, p\right]$ has a non-trivial automorphism if and only if $g_{i}=1$ and $n_{i}=0$, we conclude that a general stable curve of the form $\left[C_{1} \cup C_{2}, x_{1}, \ldots, x_{n}\right]$ admits a non-trivial automorphism if and only if either $C_{1}$ or $C_{2}$ is an elliptic tail without marked points.

The following statement should be compared to [11, Theorem 2.3].

Theorem 6.5. If $g+n \geq 4$, then the coarse moduli space $\bar{M}_{g, n}$ does not have non-trivial locally trivial deformations.

Proof. Let $c: \overline{\mathcal{M}}_{g, n} \rightarrow \overline{\mathcal{M}}_{g, n}^{\text {can }}$ the structure map on the canonical stack. Let $f: Y=$ $\bar{M}_{1,1} \times \bar{M}_{g-1, n+1} \rightarrow \bar{M}_{g, n}$ be the natural attaching morphism. Since $g+n \geq 4$, by Lemma 6.4 we have that $f(Y)$ is the locus in $\bar{M}_{g, n}$ where $c: \overline{\mathcal{M}}_{g, n} \rightarrow \overline{\mathcal{M}}_{g, n}^{\text {can }}$ is not an isomorphism. Now, on $Y$ we have an exact sequence

$$
0 \mapsto T_{Y} \rightarrow f^{*} T_{\overline{\mathcal{M}}_{g, n}} \rightarrow L \mapsto 0
$$

where $L \cong \psi_{1}^{\vee} \otimes \psi_{n+1}^{\vee}$. Since the general curve parametrized by $Y$ has two automorphisms, the line bundle $L$ fits in the following exact sequence as well

$$
0 \mapsto C_{*} T_{\overline{\mathcal{M}}_{g, n}} \rightarrow T_{\overline{\mathcal{M}}_{g, n}^{\mathrm{can}}} \rightarrow C_{*} f_{*} L^{\otimes 2} \mapsto 0
$$

Since $L^{\vee} \cong \psi_{1} \otimes \psi_{n+1}$ is big and nef [11, Theorem A.1] yields

$$
H^{0}\left(\overline{\mathcal{M}}_{g, n}^{\text {can }}, C_{*} f_{*} L^{\otimes 2}\right)=H^{1}\left(\overline{\mathcal{M}}_{g, n}^{\text {can }}, C_{*} f_{*} L^{\otimes 2}\right)=0,
$$

which in turns implies $H^{1}\left(\overline{\mathcal{M}}_{g, n}^{\text {can }}, T_{\overline{\mathcal{M}}_{g, n}^{\text {can }}}\right) \cong H^{1}\left(\overline{\mathcal{M}}_{g, n}^{\text {can }}, C_{*} T_{\overline{\mathcal{M}}_{g, n}}\right) \cong H^{1}\left(\overline{\mathcal{M}}_{g, n}, T_{\overline{\mathcal{M}}_{g, n}}\right)$. To conclude, it is enough to recall that the stack $\overline{\mathcal{M}}_{g, n}$ is rigid and to apply Lemma 6.3 with $\mathcal{X}=\overline{\mathcal{M}}_{g, n}^{\text {can }}$ and $X=\bar{M}_{g, n}$. 


\subsection{Singularities of $\bar{M}_{g, n}$}

We denote again by $\Delta_{1}$ the image in $\bar{M}_{g, n}$ of $\bar{M}_{1,1} \times \bar{M}_{g-1, n+1}$, the divisor parametrizing curves with elliptic tails. We introduce the following notation for codimension 2, that is of maximal dimension, irreducible components of the singular locus of $\bar{M}_{g, n}$ :

- $Z_{i}$ the image of $\left[E_{i}\right] \times \bar{M}_{g-1, n+1} \subset \Delta_{1}$ for $i=4,6$, the codimension-2 loci where the elliptic tail has four and six automorphisms, respectively.

- $\quad Y$ the locus parametrizing reducible curves $E \cup C$ where $E$ is an elliptic curve with a marked point which is fixed by the elliptic involution, and $C$ is a curve of genus $g-1$ with $n-1$ marked points.

- $\quad W$ the locus parametrizing reducible curves $C_{1} \cup C_{2}$ where $C_{1}$ and $C_{2}$ are of genus 2 and $g-2$, respectively, the marked points are on $C_{2}$, and $C_{1} \cap C_{2}$ is a fixed point of the hyperelliptic involution on $C_{1}$.

Definition 6.6. A variety $X$ which, étale locally at a general point of its reduced singular locus $Z$, has type $\frac{1}{n}\left(a_{1}, a_{2}, 0, \ldots, 0\right)$ will be said to have a transversal $\frac{1}{n}\left(a_{1}, a_{2}\right)$ singularity along $Z$.

Proposition 6.7. If $g+n>4$, then the only codimension-2 irreducible components of Sing $\left(\bar{M}_{g, n}\right)$ are $Z_{4}, Z_{6}, Y$, and $W$. Each component contains dense open subsets, denoted by a superscript zero, with complement of codimension at least 2 such that $\bar{M}_{g, n}$ has transversal $A_{1}$ singularities along $Z_{4}^{0}, Y^{0}$ and $W^{0}$, and transversal $\frac{1}{3}(1,1)$ singularities along $Z_{6}^{0}$.

Proof. By [30, Proposition 1] under our numerical hypothesis any component of $\operatorname{Sing}\left(M_{g, n}\right)$ has codimension at least 3 in $M_{g, n}$. By Lemma 6.4 the divisor $\Delta_{1}$ is the only boundary divisor whose general point corresponds to a curve with non-trivial automorphisms. There are 2 ways of producing loci of codimension two in $\bar{M}_{g, n}$ parametrizing curves with extra automorphisms in $\Delta_{1}$. Namely,

(a) we may add another elliptic tail,

(b) we consider the loci $Z_{i}=\left[E_{i}\right] \times \bar{M}_{g-1, n+1}$ for $i=4,6$.

Now, note that the automorphism group of the general curve parametrized by $(a)$ is generated by pseudo-reflections. Therefore, this locus is not singular for $\bar{M}_{g, n}$.

Any other component must be properly contained in a different boundary divisor, hence must be induced by a codimension-1 stratum with non-trivial automorphisms in 
some $M_{g_{1}, n_{1}+1}$ with $\left(g_{1}, n_{1}\right) \neq(1,0)$. There are exactly two such strata, for $\left(g_{1}, n_{1}+1\right)=$ $(1,2)$ and $(2,1)$, yielding the components $Y$ and $W$.

The automorphism groups of a general curve parametrized by $Z_{4}, Z_{6}, Y$ and $W$ are not generated by pseudo-reflections. We conclude that the codimension- 2 components of Sing $\left(\bar{M}_{g, n}\right)$ are exactly $Z_{4}, Z_{6}, Y$, and $W$.

Furthermore, there is just one way to produce a divisor in $Z_{i}$ parametrizing curves with extra automorphisms, namely adding another elliptic tail; this however generates a pseudo-reflection. We conclude the locus in $Z_{i}$ where the singularities are different from the generic point has codimension at least 2 . The same argument applies to $Y$ and $W$.

The finite quotient singularities in $Y^{0}$ and $W^{0}$ are both produced by automorphisms of order 2. Therefore, these singularities are forced to be of type $A_{1}$.

The type of the singularities along the $Z_{i}^{0 \prime}$ s is the same for any $g \geq 1, n \geq 2$. Therefore, by Lemma 5.1 and the second part of Proposition 5.2 we have that $Z_{4}^{0}$ is a singularity of type $\frac{1}{2}(1,1,0, \ldots, 0)$, or transversal $A_{1}$, and $Z_{6}^{0}$ is a singularity of type $\frac{1}{3}(1,1,0, \ldots, 0)$ or transversal $\frac{1}{3}(1,1)$.

\subsection{Deformation of varieties with transversal $A_{1}$ singularities}

In this section, we assume that the characteristic of the ground field is different from two. Let $\mathcal{X}$ be a smooth Deligne-Mumford stack, and assume that its inertia stack is the disjoint union of $\mathcal{X}$ and of a smooth stack $\mathcal{Z}$, mapping isomorphically to its image in $\mathcal{X}$, which we also denote by $\mathcal{Z}$. Let $\varepsilon: \mathcal{X} \rightarrow X$ be the morphism to the coarse moduli space, which we assume is a scheme. Let $\bar{\varepsilon}: \mathcal{Z} \rightarrow Z$ be the morphism to the coarse moduli space, and assume that $\bar{\varepsilon}$ is a $\mu_{2}$-gerbe.

An example can be constructed as follows: let $Z$ be a non-singular variety, and $F$ a rank-2 vector bundle on $Z$. Let $\mu_{2}$ act on $F$ by scalar multiplication. Then, $\mathcal{X}=\left[F / \mu_{2}\right]$ and $\mathcal{Z}=Z \times B \mu_{2}$ satisfy the assumptions.

In this case, $X$ is a variety with transversal $A_{1}$, or transversal $\frac{1}{2}(1,1)$ singularities along $Z$, that is étale locally, the inclusion $i: Z \rightarrow X$ looks like $Z=Z \times\{0\} \rightarrow$ $Z \times \operatorname{Spec} K[x, y, z] /\left(x y-z^{2}\right)$. Conversely, if $X$ has transversal $A_{1}$ singularities along $Z$, then such an $\varepsilon: \mathcal{X} \rightarrow X$ exists, it is the canonical stack, and is unique up to unique isomorphism, while $\mathcal{Z}=\varepsilon^{-1}(Z)_{\text {red }}$.

Let $N:=N_{\mathcal{Z} / \mathcal{X}}$ be the normal bundle of $\mathcal{Z}$ in $\mathcal{X}$; there exist a, unique up to isomorphism, rank-3 vector bundle $V$ on $Z$ and a, unique up to isomorphism, line bundle $L$ on $Z$, such that $\bar{\varepsilon}^{*}(E)=\operatorname{Sym}^{2} N$ and $\bar{\varepsilon}^{*}(L)=\operatorname{det} N$. Let $\pi: V \rightarrow Z$ be the projection, and 
let $\bar{L}:=\pi^{*} L$. Let $C$ be the coarse moduli space of $N ; C$ has transversal $A_{1}$ singularities along $Z$, it is easy to see that $C=C_{Z / X}$, the normal cone of $Z$ in $X$, but we will not need this.

Lemma 6.8. There is a natural embedding of $C$ in $V$ as the scheme-theoretic zero section of $\bar{L}^{\otimes 2}$.

Proof. Let us consider the principal GL(2) bundle $P \rightarrow \mathcal{Z}$ associated with $N$, and denote by $N_{P}$ the pull-back of $N$ to $P$, which is canonically trivial as each point of $P$ corresponds to a basis of a fiber of $N$. A basis $n_{1}, n_{2}$ of $N_{z}$ induces a basis $n_{11}, n_{12}, n_{22}$ of $E_{P}$ hence a trivialization, with coordinates $x_{11}, x_{12}, x_{22}, x_{i j}=n_{i} n_{j}$. The rank-1 tensors are given by the equation $f=x_{11} x_{22}-x_{12}^{2}$. It is easy to see that the section $f\left(n_{1} \wedge n_{2}\right)^{\otimes 2}$ of $L_{P}$ is $G L(2)$-invariant.

Theorem 6.9. In our assumptions we have $\mathcal{E} \mathrm{xt}^{1}\left(\Omega_{X}, \mathcal{O}_{X}\right)=i_{*} \bar{L}^{\otimes 2}$, where $i: Z \rightarrow X$ is the natural embedding.

Proof. Assume first that $\mathcal{X}=N$, hence $X=C$; then the result immediately follows from Lemma 6.8 , by applying the functor $\mathcal{H o m}\left(-, \mathcal{O}_{X}\right)$ to the exact sequence

$$
0 \mapsto \mathcal{O}_{V}(-C)_{\mid C} \rightarrow \Omega_{V \mid C} \rightarrow \Omega_{C} \mapsto 0
$$

For the general case, we first prove that the sheaf is the push-forward of a line bundle on $Z$, since this statement is étale local hence we reduce to the previous case. To identify the line bundle, we use the degeneration to the normal cone of the inclusion $\mathcal{Z} \rightarrow \mathcal{X}$; we get a one-parameter family of line bundles on $Z$ where the general one is $\mathcal{E} \mathrm{xt}^{1}\left(\Omega_{X}, \mathcal{O}_{X}\right)$ and the special one is $\bar{L}^{\otimes 2}$; we conclude that they are equal since the Picard scheme of $Z$ is separated.

\subsection{Deformation of varieties with transversal $\frac{1}{3}(1,1)$ singularity}

In this section, we assume the characteristic to be different from two and three. Again we start with a special case. Assume that $Z$ is a smooth variety, and $F$ the total space of a rank- 2 vector bundle on $Z$. Let the group $\mu_{3}$ act on $F$ by scalars, and let $\mathcal{X}=\left[F / \mu_{3}\right]$ and $\varepsilon: \mathcal{X} \rightarrow X$ be the morphism to the coarse moduli space, which is an affine variety, and indeed a cone over $Z$.

Assume now that $F=L_{1} \oplus L_{2}$ with $L_{i}$ line bundles on $Z$. Write $L_{i j}:=L_{1}^{\otimes i} \otimes L_{2}^{\otimes j} \in$ $\operatorname{Pic}(Z)$. Let $V=\operatorname{Sym}^{3} F=L_{03} \oplus L_{12} \oplus L_{21} \oplus L_{30}$. Let $\pi: V \rightarrow Z$ be the projection, and write 
$\bar{L}_{i j}:=\pi^{*}\left(L_{i j}\right)$. Finally, let $\sigma$ be the tautological section of $\pi^{*} V$, and write $\sigma=\left(s_{0}, s_{1}, s_{2}, s_{3}\right)$ with $s_{i}$ a section of $\bar{L}_{i, 3-i}$.

There is a natural embedding $X \rightarrow V$, where $X$ parametrizes rank-1 tensors in $\operatorname{Sym}^{3}(F)$. Write $\widetilde{L}_{i j}:=\bar{L}_{i j \mid X}$.

Lemma 6.10. There is an exact sequence of coherent sheaves on $X$

$$
\widetilde{L}_{54}^{\vee} \oplus \widetilde{L}_{45}^{\vee} \rightarrow \widetilde{L}_{24}^{\vee} \oplus \widetilde{L}_{33}^{\vee} \oplus \widetilde{L}_{42}^{\vee} \rightarrow \widetilde{L}_{03}^{\vee} \oplus \widetilde{L}_{12}^{\vee} \oplus \widetilde{L}_{21}^{\vee} \oplus \widetilde{L}_{03}^{\vee} \oplus \pi^{*} \Omega_{Z \mid X} \rightarrow \Omega_{X} \mapsto 0,
$$

where the first map $\alpha$ is given by the matrix

$$
\left(\begin{array}{ll}
s_{3} & s_{2} \\
s_{2} & s_{1} \\
s_{1} & s_{0}
\end{array}\right)
$$

and the second map $\beta$ is given by the matrix

$$
\left(\begin{array}{ccc}
s_{2} & -s_{3} & 0 \\
-2 s_{1} & s_{2} & s_{3} \\
s_{0} & s_{1} & -2 s_{2} \\
0 & -s_{1} & s_{0}
\end{array}\right)
$$

Proof. Note that $X$ is the scheme-theoretic zero locus in $V$ of the section $\left(s_{0} s_{2}-s_{1}^{2}, s_{1} s_{2}-\right.$ $s_{0} s_{3}, s_{1} s_{3}-s_{2}^{2}$ ) of $\bar{L}_{24} \oplus \bar{L}_{33} \oplus \bar{L}_{42}$. The rest of the statement is elementary homological algebra.

Proposition 6.11. The sheaf $\mathcal{E} \mathrm{xt}^{1}\left(\Omega_{X}, \mathcal{O}_{X}\right)$ is isomorphic to $i_{*}\left(L_{21} \oplus L_{12}\right)=i_{*}(F) \otimes \operatorname{det} F$.

Proof. Let $\alpha$ be the map in Lemma 6.10. Then $\alpha^{\vee}$ is the second map in an exact sequence

$$
\widetilde{L}_{21} \oplus \widetilde{L}_{12} \oplus \widetilde{L}_{03} \oplus \widetilde{L}_{30} \oplus \widetilde{L}_{21} \oplus \widetilde{L}_{11} \rightarrow \widetilde{L}_{24} \oplus \widetilde{L}_{33} \oplus \widetilde{L}_{42} \rightarrow \widetilde{L}_{54} \oplus \widetilde{L}_{45}
$$

where the first map $\gamma$ is given by the matrix

$$
\left(\begin{array}{cccccc}
s_{0} & s_{1} & s_{2} & 0 & 0 & 0 \\
-s_{1} & -s_{2} & -s_{3} & s_{0} & s_{1} & s_{2} \\
0 & 0 & 0 & -s_{1} & -s_{2} & -s_{3}
\end{array}\right)
$$

We conclude by observing that $\mathcal{I}_{Z / X} \cdot \mathcal{E x t}^{1}\left(\Omega_{X}, \mathcal{O}_{X}\right)=0$ and by comparing the two exact sequences. 
Recall that by Definition 6.6 a variety $X$ which, étale locally near each point of its reduced singular locus $Z$, has type $\frac{1}{3}(1,1,0, \ldots, 0)$ will be said to have a transversal $\frac{1}{3}(1,1)$ singularity along $Z$. We denote by $\varepsilon: \mathcal{X} \rightarrow X$ the associated canonical stack. Let $\mathcal{Z}:=\varepsilon^{-1}(Z)_{\text {red }}$ and $\bar{\varepsilon}:=\left.\varepsilon\right|_{\mathcal{Z}}: \mathcal{Z} \rightarrow Z$, which is a $B \mu_{3}$-gerbe.

Corollary 6.12. Assume that $X$ has a transversal $\frac{1}{3}(1,1)$ singularity along $Z$, and moreover that there exist line bundles $L_{1}, L_{2}$ on $\mathcal{Z}$ such that $N:=N_{\mathcal{Z} / \mathcal{X}}$ fits into an exact sequence

$$
0 \mapsto \bar{L}_{1} \rightarrow N \rightarrow \bar{L}_{2} \mapsto 0
$$

Write $\bar{L}_{i j}:=\bar{L}_{1}^{\otimes i} \otimes \bar{L}_{2}^{\otimes j}$; when $i+j$ is a multiple of three, write $L_{i j}$ for the line bundle on $Z$ whose pull-back to $\mathcal{Z}$ is $\bar{L}_{i j}$. If $H^{0}\left(Z, L_{12}\right)=H^{0}\left(Z, L_{21}\right)=0$, then we have $H^{0}\left(X, \mathcal{E} \mathrm{Xt}^{1}\left(\Omega_{X}, \mathcal{O}_{X}\right)\right)=0$.

Proof. Since étale locally we are in the situation of the Proposition 6.11, we have $\mathcal{E x t}^{1}\left(\Omega_{X}, \mathcal{O}_{X}\right)=i_{*} E$ where $E$ is a rank-2 vector bundle on $Z$ and $i: Z \rightarrow X$ is the inclusion. We can again degenerate the inclusion $\mathcal{Z} \rightarrow \mathcal{X}$ to the normal cone, this degenerates $E$ to some other line bundle $E^{\prime}$, and by semi-continuity it will be enough to show that $E^{\prime}$ does not have global sections on $Z$. We are now assuming that $\mathcal{X}=N$; again we can degenerate $N$ to $\bar{\varepsilon}^{*} L_{1} \oplus \bar{\varepsilon}^{*} L_{2}$, hence we are now in the set-up of Proposition 6.11 , and $E^{\prime}$ degenerates to $L_{12} \oplus L_{21}$. By our assumptions the latter has no non-zero global sections.

We are ready to prove the main result of this section.

Theorem 6.13. If $g+n>4$, over an algebraically closed field of characteristic zero, the coarse moduli space $\bar{M}_{g, n}$ is rigid.

Proof. By Theorem 6.5 we know that $\bar{M}_{g, n}$ does not have locally trivial deformations. To conclude it is enough to prove that $H^{0}\left(\bar{M}_{g, n}, \mathcal{E} \operatorname{xt}^{1}\left(\Omega_{\bar{M}_{g, n}}, \mathcal{O}_{\bar{M}_{g, n}}\right)\right)=0$.

Now, $\mathcal{E} \mathrm{xt}^{1}\left(\Omega_{\bar{M}_{g, n}}, \mathcal{O}_{\bar{M}_{g, n}}\right)$ is a coherent sheaf supported on $\operatorname{Sing}\left(\bar{M}_{g, n}\right)$. Let $S_{3} \subset$ $\operatorname{Sing}\left(\bar{M}_{g, n}\right)$ be the union of all irreducible components which have codimension $\geq 3$. By Remark 6.2 there are no sections of $\mathcal{E x t}^{1}\left(\Omega_{\bar{M}_{g, n},} \mathcal{O}_{\bar{M}_{g, n}}\right)$ supported on $S_{3}$. By Proposition 6.7 we know the codimension-2 components of $\bar{M}_{g, n}$ are $Z_{6}, Z_{4}, Y$, and $W$, and again there are no sections supported on $Z_{6} \backslash Z_{6}^{0}$, since by Proposition $6.7 Z_{6}^{0}$ has codimension at least 2 in $Z_{6}$, and similarly for $Z_{4}, Y$, and $W$. Thus, it will be enough to show $H^{0}\left(Z_{6}^{0}, \mathcal{E x t}^{1}\left(\Omega_{\bar{M}_{g, n}}, \mathcal{O}_{\bar{M}_{g, n}}\right)_{\mid Z_{6}^{0}}\right)=0$ and similarly for $Z_{4}^{0}, Y^{0}$, and $W^{0}$. We will give in detail the proof for $Z_{6}^{0}$ and sketch the other cases. 
Let $\mathcal{Z}_{6}$ be the image in $\overline{\mathcal{M}}_{g, n}$ of $\left[E_{6}\right] \times \overline{\mathcal{M}}_{g-1, n+1}=B \mu_{6} \times \overline{\mathcal{M}}_{g-1, n+1}$; let $\mathcal{Z}_{6}^{\text {can }}$ be its image in $\overline{\mathcal{M}}_{g, n}^{\text {can }} ; \mathcal{Z}_{6}^{\text {can }}$ is the inverse image of $Z_{6}$ in the canonical stack, with the reduced induced structure. Denote as usual by $\Delta_{1} \subset \overline{\mathcal{M}}_{g, n}$ the divisor of rational tails, and let $\Delta_{1}^{\text {can }}$ be its image in $\overline{\mathcal{M}}_{g, n}^{\text {can }}$. Consider the commutative diagram

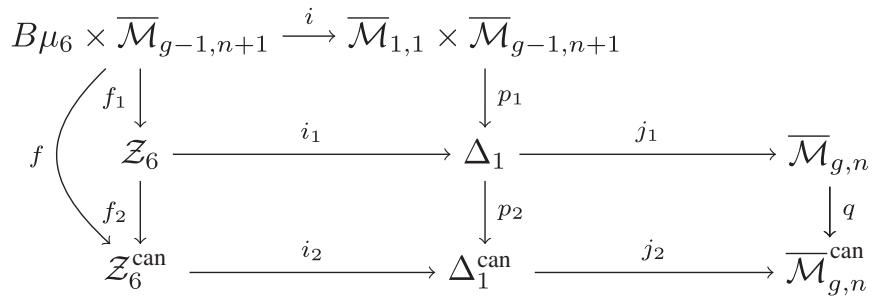

where the horizontal arrows are closed embeddings, and the vertical ones are proper, finite morphisms. Let $U$ be the inverse image in $B \mu_{6} \times \overline{\mathcal{M}}_{g-1, n+1}$ of $Z_{6}^{0}$; at all points of $U$, and their images, all the stacks of the diagram are smooth and all vertical maps are bijective on points; we have that $N_{i \mid U}=f^{*} N_{i_{2} \mid U}$ while $f^{*} i_{2}^{*} N_{j_{2} \mid U}=f_{1}^{*} i_{1}^{*} N_{j_{1} \mid U}^{\otimes 2}$ since the morphism $p_{1}$ is ramified along $\Delta_{1}$ of order 2 . Now we have that $N_{i}=p r_{1}^{*} T_{\bar{M}_{1,1} \mid B \mu_{6}}$ while $p_{1}^{*} N_{j_{1}}=p r_{1}^{*} \psi_{1}^{\vee} \otimes p r_{2}^{*} \psi_{n+1}^{\vee}$. To prove that $H^{0}\left(Z_{6}^{0}, \mathcal{E} \operatorname{xt}^{1}\left(\Omega_{\bar{M}_{g, n}}, \mathcal{O}_{\bar{M}_{g, n}}\right)_{\mid Z_{6}^{0}}\right)=0$ is equivalent, in view of Corollary 6.12 together with the fact that $f$ over $Z_{6}^{0}$ is finite and bijective on points, to show that

$$
H^{0}\left(U, N_{i}^{\otimes 2} \otimes\left(p r_{1}^{*} \psi_{1}^{\vee} \otimes p r_{2}^{*} \psi_{n+1}^{\vee}\right)_{\mid U}^{\otimes 2}\right)=H^{0}\left(U, N_{i} \otimes\left(p r_{1}^{*} \psi_{1}^{\vee} \otimes p r_{2}^{*} \psi_{n+1}^{\vee}\right)_{\mid U}^{\otimes 4}\right)=0
$$

Since the complement of $U$ in $B \mu_{6} \times \overline{\mathcal{M}}_{g-1, n+1}$ has codimension 2, it is enough to prove that

$$
\begin{array}{r}
H^{0}\left(B \mu_{6} \times \overline{\mathcal{M}}_{g-1, n+1}, N_{i}^{\otimes 2} \otimes\left(p r_{1}^{*} \psi_{1}^{\vee} \otimes p r_{2}^{*} \psi_{n+1}^{\vee}\right)^{\otimes 2}\right)= \\
H^{0}\left(B \mu_{6} \times \overline{\mathcal{M}}_{g-1, n+1}, N_{i} \otimes\left(p r_{1}^{*} \psi_{1}^{\vee} \otimes p r_{2}^{*} \psi_{n+1}^{\vee}\right)^{\otimes 4}\right)
\end{array}
$$

are zero. By [11, Sections 2, 3] psi-classes are nef and big. To conclude, it is enough to use Kodaira vanishing [11, Theorem A.1].

For the components $Z_{4}^{0}, Y^{0}, W^{0}$ the relevant commuting diagram is obtained in the same way (by taking images in $\overline{\mathcal{M}}_{g, n}$ in the second row and in $\overline{\mathcal{M}}_{g, n}^{\text {can }}$ in the third row), but taking as first row the following embeddings:

- for $Z_{4}^{0}, B \mu_{4} \times \overline{\mathcal{M}}_{g-1, n+1} \rightarrow \overline{\mathcal{M}}_{1,1} \times \overline{\mathcal{M}}_{g-1, n+1}$ where $B \mu_{4}$ maps to the point [E $\left.E_{4}\right]$; 
- for $Y^{0}, A \times \overline{\mathcal{M}}_{g-1, n} \rightarrow \overline{\mathcal{M}}_{1,2} \times \overline{\mathcal{M}}_{g-1, n+2}$ where $A \subset \overline{\mathcal{M}}_{1,2}$ is the closure of the locus parametrizing triples $\left(E, p_{1}, p_{2}\right)$ where $\left(E, p_{1}\right)$ is a smooth elliptic curve and $p_{2}$ is a 2 -torsion point in $E$;

- for $W^{0}, A^{\prime} \times \overline{\mathcal{M}}_{g-2, n+1} \rightarrow \overline{\mathcal{M}}_{2,1} \times \overline{\mathcal{M}}_{g-2, n+1}$ where $A^{\prime} \subset \overline{\mathcal{M}}_{2,1}$ is the closure of the locus parametrizing triples $\left(C, p_{1}\right)$ where $C$ is a smooth genus-2 curve and $p_{1}$ is a point fixed by the hyperellitpic involution.

We argue in an analogous way and use Theorem 6.9 instead of Corollary 6.12, and conclude as before using the fact that $\psi$ classes are nef and big.

\section{Automorphisms of $\bar{M}_{g, n}$ Over an Arbitrary Field}

In this section, we will use the main results of Sections 2 and 4 to compute the automorphism groups of $\bar{M}_{g, n}$ over an arbitrary field. In order to do this we will apply the theory developed in Section 2 taking $A=W(K)$, that is, the ring of Witt vectors over $K$, see [35] for details.

For our purposes it is enough to keep in mind that $W(K)$ is a discrete valuation ring with a closed point $x \in \operatorname{Spec}(W(K))$ with residue field $K$, and a generic point $\xi \in$ $\operatorname{Spec}(W(K))$ with residue field of characteristic zero.

Proposition 7.1. Assume that Aut $\left(\bar{M}_{g, n}^{\bar{K}}\right) \cong S_{n}$ where $K$ is any field of characteristic zero. Then $\operatorname{Aut}\left(\bar{M}_{g, n}^{K}\right) \cong S_{n}$. Furthermore, if $H^{0}\left(\bar{M}_{g, n}^{K}, T_{\bar{M}_{g, n}^{K}}\right)=0$ and $\bar{M}_{g, n}^{K}$ is rigid for some field $K_{p}$ of characteristic $p$ then $\operatorname{Aut}\left(\bar{M}_{g, n}^{K p}\right) \cong S_{n}$.

Proof. By Lemma 2.2 there is an injective morphism of groups

$$
\chi: \operatorname{Aut}\left(\bar{M}_{g, n}^{K}\right) \rightarrow \operatorname{Aut}\left(\bar{M}_{g, n}^{\bar{K}}\right)
$$

The hypothesis Aut $\left(\bar{M}_{g, n}^{\bar{K}}\right) \cong S_{n}$ forces $\chi$ to be an isomorphism. Let $A$ be a ring of Witt vectors $W\left(K_{p}\right)$ of $K_{p}$ and $K$ its quotient field. Recall that $\operatorname{char}(K)=0$. By Theorem 2.6 we have an injective morphism of groups

$$
\chi_{p}: \operatorname{Aut}\left(\bar{M}_{g, n}^{K p}\right) \rightarrow \operatorname{Aut}\left(\bar{M}_{g, n}^{K}\right) .
$$

By the first part of the proof $\operatorname{Aut}\left(\bar{M}_{g, n}^{K}\right) \cong S_{n}$, and $\chi_{p}$ is an isomorphism.

Finally, we can prove analogue of Theorem 7.1 for the stack $\overline{\mathcal{M}}_{g, n}$. 
Proposition 7.2. Assume that Aut $\left(\bar{M}_{g, n}^{\bar{K}}\right) \cong S_{n}$ and $H^{0}\left(\overline{\mathcal{M}}_{g, n}^{K}, T_{\overline{\mathcal{M}}_{g, n}^{K}}\right)=0$ for $K$ any field of characteristic zero. If for some field $K_{p}$ of characteristic $p$ the stack $\overline{\mathcal{M}}_{g, n}^{K p}$ is rigid then $\operatorname{Aut}\left(\overline{\mathcal{M}}_{g, n}^{K p}\right) \cong S_{n}$.

Proof. Let again $A$ be a ring of Witt vectors $W(K)$ of $K_{p}$ with residue field $K$. Let $\phi \in \operatorname{Aut}\left(\overline{\mathcal{M}}_{g, n}^{K p}\right)$ be an automorphism. As in the proof of Theorem 7.1, the rigidity assumption and semi-continuity imply that $H^{0}\left(\overline{\mathcal{M}}_{g, n}^{K_{p}}, T_{\overline{\mathcal{M}}_{g, n}^{K p}}\right)=0$ hence by Lemma $2.3 \phi$ is the restriction of a unique $\phi_{i} \in \operatorname{Aut}\left(\overline{\mathcal{M}}_{g, n}^{A_{i}} / A_{i}\right)$ for every $i \geq 1$. By Proposition 2.7 the automorphism $\phi_{i}$ induces an automorphism $\tilde{\phi}_{i} \in \operatorname{Aut}\left(\bar{M}_{g, n}^{A_{i}} / A_{i}\right)$ such that the restriction of $\tilde{\phi}_{i+1}$ to $\bar{M}_{g, n}^{A_{i}}$ is $\tilde{\phi}_{i}$. Thus, reasoning as in Theorem 2.6 there exists a unique automorphism $\tilde{\phi}_{A} \in \operatorname{Aut}\left(\bar{M}_{g, n}^{A} / A\right)$ inducing $\tilde{\phi}_{i}$ for any $i \geq 0$. Let $\tilde{\phi}_{\mid \bar{M}_{g, n}^{K}}$ be the restriction of $\tilde{\phi}$ to $\bar{M}_{g, n}^{K}$. This construction yields an injective morphism of groups

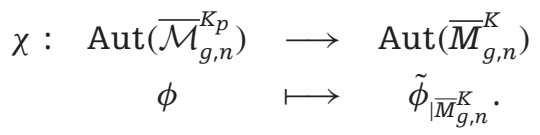

Now, to conclude it is enough to argue as in the proof of Proposition 7.1.

Now, we are ready to prove the main result of this section.

Theorem 7.3. Let $K$ be any field then

$$
\operatorname{Aut}\left(\bar{M}_{0, n}\right) \cong S_{n}
$$

for any $n \geq 5$. Furthermore, if $K$ is any field with $\operatorname{char}(K) \neq 2$ then

$$
\operatorname{Aut}\left(\overline{\mathcal{M}}_{g, n}\right) \cong \operatorname{Aut}\left(\bar{M}_{g, n}\right) \cong S_{n}
$$

for any $(g, n) \neq(2,1)$ such that $2 g-2+n \geq 3$ and $n \geq 1$. Finally, over any field of characteristic zero we have that $\operatorname{Aut}\left(\overline{\mathcal{M}}_{2,1}\right) \cong \operatorname{Aut}\left(\bar{M}_{2,1}\right)$ is trivial, and $\operatorname{Aut}\left(\overline{\mathcal{M}}_{g}\right) \cong \operatorname{Aut}\left(\bar{M}_{g}\right)$ is trivial for any $g \geq 2$.

Proof. Let us begin with the genus zero case. If $K$ is any field of characteristic zero, the statement follows from Theorem A.1 and the first part of Proposition 7.1. If $K$ is any field of positive characteristic then by Theorem $4.1 \bar{M}_{0, n}^{K}$ is rigid and $H^{0}\left(\bar{M}_{0, n}^{K}, T_{\bar{M}_{0, n}^{K}}\right)$ for $n \geq 5$. Now, to conclude it is enough to apply the second part of Proposition 7.1. 
The statement for $(g, n) \neq(2,1), 2 g-2+n \geq 3$, and $n \geq 1$ follows by Propositions 7.1, 2.7 together with Theorem A.2. Finally, Propositions 7.1, 2.7, and Remark A.3 yield the result for $(g, n)=(2,1)$ and $g \geq 2, n=0$.

By [31, Proposition 4.2.8] any group scheme over a field of characteristic zero is reduced. However, in positive characteristic a group scheme is not necessarily reduced. For instance, in [20] there are examples of non-reduced Picard schemes. By Theorem 7.3 we know that over any field of positive characteristic $\operatorname{Aut}\left(\bar{M}_{0, n}\right) \cong S_{n}$ for any $n \geq 5$. A priori this is not enough to conclude that $\operatorname{Aut}\left(\bar{M}_{0, n}\right)$ is reduced. Anyway we have the following proposition.

Proposition 7.4. Let $K$ be a field of positive characteristic. Then, $\operatorname{Aut}\left(\bar{M}_{0, n}\right)$ is reduced.

Proof. The tangent space of Aut $\left(\bar{M}_{0, n}\right)$ at the identity is given by

$$
T_{[I d]} \operatorname{Aut}\left(\bar{M}_{0, n}\right) \cong H^{0}\left(\bar{M}_{0, n}, T_{\bar{M}_{0, n}}\right) .
$$

If $n \geq 5$, by Theorem 4.1 we have $\operatorname{dim}\left(T_{[I d]} \operatorname{Aut}\left(\bar{M}_{0, n}\right)\right)=h^{0}\left(\bar{M}_{0, n}, T_{\bar{M}_{0, n}}\right)=0$. So Aut $\left(\bar{M}_{0, n}\right)$ is reduced. If $n=4$, then $\operatorname{Aut}\left(\bar{M}_{0,4}\right) \cong \operatorname{PGL}(2)$ which is reduced as well.

\section{Appendix Automorphisms of $\bar{M}_{g, n}$ Over Algebraically Closed Fields}

In this appendix, we essentially extend the main result of [23] on the automorphisms of $\bar{M}_{g, n}$ and $\overline{\mathcal{M}}_{g, n}$ working on an algebraically closed field $K$ with $\operatorname{char}(K) \neq 2$.

Let us begin by discussing the case $g=0$. In [2] Bruno and Mella, thanks to Kapranov's work [15], managed to translate issues on the moduli space $\bar{M}_{0, n}$ in terms of classical projective geometry of $\mathbb{P}^{n-3}$. Studying linear systems on $\mathbb{P}^{n-3}$ with particular base loci they derived a theorem on the automorphisms of $\bar{M}_{0, n}$ over an algebraically closed field of characteristic zero.

Theorem A.1. [2, Theorem 3] Let $K$ be an algebraically closed field of characteristic zero. Then the automorphism group of $\bar{M}_{0, n}$ is isomorphic to $S_{n}$ for any $n \geq 5$.

In [23], a similar result is proven for $\bar{M}_{g, n}$ over the field $\mathbb{C}$ of complex numbers. However, the proof of [23, Theorem 3.10] works over any algebraically closed field $K$ with $\operatorname{char}(K) \neq 2$. This is just because [9, Theorem 0.9] works over any algebraically 
closed field of characteristic different from two and a general $n$-pointed genus $g$ is automorphism-free if $2 g-2+n \geq 3$.

Theorem A.2. Let $K$ be an algebraically closed field with $\operatorname{char}(K) \neq 2$. If $2 g-2+n \geq 3$, $g \geq 1$ and $(g, n) \neq(2,1)$, then

$$
\operatorname{Aut}\left(\overline{\mathcal{M}}_{g, n}\right) \cong \operatorname{Aut}\left(\bar{M}_{g, n}\right) \cong S_{n}
$$

Remark A.3. By [23, Proposition 3.6, Theorem 4.4], if $K$ is algebraically closed of characteristic zero we have that $\operatorname{Aut}\left(\bar{M}_{2,1}\right) \cong \operatorname{Aut}\left(\bar{M}_{2,1}\right)$ is trivial. Furthermore, by [23, Proposition 3.5, Theorem 4.4] we have that $\operatorname{Aut}\left(\bar{M}_{g}\right) \cong \operatorname{Aut}\left(\bar{M}_{g}\right)$ is trivial for any $g \geq 2$. The proofs are based on Royden's theorem [27, Theorem 6.1] which works just over an algebraically closed of characteristic zero .

Remark A.4. Let $K$ be an algebraically closed field with $\operatorname{char}(K) \neq 2,3$. By [23, Proposition 4.5] we have $\operatorname{Aut}\left(\overline{\mathcal{M}}_{1,2}\right)$ is trivial. Indeed, if $\operatorname{char}(K)=0$ by [11, Theorem 2.1] $T_{[I d]} \operatorname{Aut}\left(\overline{\mathcal{M}}_{1,2}\right) \cong H^{0}\left(\overline{\mathcal{M}}_{1,2}, T_{\overline{\mathcal{M}}_{1,2}}\right)=0$. Finally, since $\bar{M}_{1,1} \cong \mathbb{P}^{1}$ and $\overline{\mathcal{M}}_{1,1} \cong \mathbb{P}(4,6)$ we have $\operatorname{Aut}\left(\bar{M}_{1,1}\right) \cong \operatorname{PGL}(2)$, while $\operatorname{Aut}\left(\overline{\mathcal{M}}_{1,1}\right) \cong K^{*}$.

\section{Acknowledgments}

This work was done while A.M. was a Post-Doctorate at IMPA, funded by CAPES-Brazil. We would like to thank Aise Johan de Jong and Massimiliano Mella for bringing this subject to our attention and for many helpful comments, Paul Hacking for pointing us out [5], and Nicola Pagani for useful discussion about the singularities of coarse moduli spaces.

\section{References}

[1] Artin, M. Lectures on Deformations of Singularities. Bombay: Tata Institute of Fundamental Research, Bombay, 1976.

[2] Bruno, A., and M. Mella. "The automorphism group of $\bar{M}_{0, n}$. " Journal of the European Mathematical Society 15, no. 3 (2013): 949-68.

[3] Cornalba, M. "On the locus of curves with automorphisms." Annali di Matematica Pura ed Applicata 149, no. 1 (1987): 135-51.

[4] Cox, D. A., J. B. Little, and H. K. Schenck. Toric Varieties. Graduate Studies in Mathematics. Providence, RI, USA: American Mathematical Society, 2011.

[5] Deligne, P., and L. Illusie. "Relèvements modulo $p^{2}$ et décomposition du complexe de de Rham." Inventiones Mathematicae 89, no. 2 (1987): 247-70.

[6] Fantechi, B. "Deformation of Hilbert Schemes of Points on a Surface." Compositio Mathematica 98 (1995): 205-17. 
[7] Fantechi, B., L. Göttsche, L. Illusie, S. Kleiman, N. Nitsure, and A. Vistoli. Fundamental Algebraic Geometry. Grothendieck's FGA Explained. Mathematical Surveys and Monographs, 123. Providence, RI: American Mathematical Society, 2005.

[8] Fantechi, B., E. Mann, and F. Nironi. "Smooth toric DM stacks." Journal für die reine und angewandte Mathematik 648 (2010): 201-44.

[9] Gibney, A., S. Keel, and I. Morrison. "Towards the ample cone of $\bar{M}_{g, n}$." Journal of the American Mathematical Society 15, no. 2 (2002): 273-94.

[10] Grothendieck, A. Cohomologie locale des faisceaux cohérents et théorèmes de Lefschetz locaux et globaux, North Holland: SGA2, 1968.

[11] Hacking, P. "The moduli space of curves is rigid." Algebra Number Theory 2, no. 7 (2008): 809-18.

[12] Hartshorne, R. Algebraic Geometry. Graduate Texts in Mathematics, no. 52. New York: Springer, 1977.

[13] Haykawa, T. "Blowing Ups of 3-dimensional Terminal Singularities." Publications of the Research Institute for Mathematical Sciences, Kyoto University 35 (1999): 515-70.

[14] Illusie, L. Complexe Cotangent et Déformations I. Springer Lecture Notes 239. Heidelberg, Berlin: Springer, 1971.

[15] Kapranov, M. "Veronese curves and Grothendieck-Knudsen moduli space $\bar{M}_{0, n}$. " Journal of Algebraic Geometry 2, no. 2 (1993): 239-62.

[16] Kapranov, M. Deformations of Moduli Spaces, 1997. Unpublished manuscript.

[17] Keel, S. "Basepoint freeness for nef and big line bundles in positive characteristic." Annals of Mathematics 2 149, no. 1 (1999): 253-86.

[18] Keel, S., and S. Mori. “Quotients by groupoids.” Annals of Mathematics 2145 (1997): 193-213.

[19] Knudsen, F. F. "The projectivity of the moduli space of stable curves II. The stacks $M_{g, n}$. " Mathematica Scandinavica 52, no. 2 (1983): 161-99.

[20] Liedtke, C. "A note on non-reduced Picard schemes." Journal of Pure and Applied Algebra 213, no. 5 (2009): 737-41.

[21] Lin, V. "Configuration spaces of $\mathbb{C}$ and $\mathbb{C P}^{1}$ : some analytic properties." (2004): MaxPlanckInstitute für Mathematik, Bonn, MPI 2003-98 (2003), 80 p. http://www.mpimbonn.mpg.de/ preblob/2266.

[22] Lin, V. "Algebraic functions, configuration spaces, Teichmüller spaces, and new holomorphically combinatorial invariants." Functional Analysis and Its Applications 45 (2011): 204-24.

[23] Massarenti, A. "The automorphism group of $\bar{M}_{g, n}$. " Journal of the London Mathematical Society 89 (2014): 131-50.

[24] Massarenti, A. "On the biregular geometry of Fulton-MacPherson configuration spaces." (2016): arXiv:1603.06991v1.

[25] Massarenti, A., and M. Mella. "On the Automorphisms of Moduli Spaces of Curves." Springer Proceedings in Mathematics and Statistics, Vol. 79, 2014.

[26] Massarenti, A., and M. Mella. "On the automorphisms of Hassett's moduli spaces." To appear on Transactions of the American Mathematical Society, (2015): arXiv:1307.6828. 
[27] Mochizuki, S. “Correspondences on hyperbolic curves." Journal of Pure and Applied Algebra 131 (1998): 227-44.

[28] Moriwaki, A. "The $\mathbb{Q}$-Picard group of the moduli space of curves in positive characteristic." International Journal of Mathematics 12 (2001): 519-34.

[29] Okawa, S., and T. Sano. "Noncommutative rigidity of the moduli stack of stable pointed curves." (2014): arXiv:1412.7060v1.

[30] Pagani, N. "Harer stability and orbifold cohomology." Pacific Journal of Mathematics 267, no. 2 (2014): 465-77.

[31] Perrin, D. "Approximation des schémas en groupes, quasi compacts sur un corps." Bulletin de la Société Mathématique de France 104, no. 3 (1976): 323-35.

[32] Prokhorov, Y. G. Lectures on Complements on Log Surfaces. MSJ Memoirs, 10. Tokyo: Mathematical Society of Japan, 2001.

[33] Royden, H. L. "Automorphisms and Isometries of Teichmüller Spaces." Advances in the Theory of Riemann Surfaces edited by L. V. Ahlfors, L. Bers, H. M. Farkas, R. C. Gunning, I. Kra, H. E. Rauch. Annals of Mathematical Studies, Princeton, NJ: Princeton Univ. Press, no. $66,1971,369-83$.

[34] Sernesi, E. "Deformations of Algebraic Schemes." Grundlehren der Mathematischen Wissenschaften. Fundamental Principles of Mathematical Sciences, Heidelberg, Berlin, 334, 2006.

[35] Witt, E. "Zyklische Körper und Algebren der Characteristik $p$ vom Grad $p^{n}$. Struktur diskret bewerteter perfekter Körper mit vollkommenem Restklassenkörper der Charakteristik $p^{n} . "$ Journal für die reine und angewandte Mathematik 176 (1936): 126-40. 